\title{
A Unifying Theory of Dark Energy, Dark Matter, and Baryonic Matter in the Positive-Negative Mass Universe Pair: Protogalaxy and Galaxy Evolutions
}

\author{
Ding-Yu Chung \\ Utica, Michigan, USA \\ Email: dy_chung@yahoo.com
}

How to cite this paper: Chung, D.-Y. (2020) A Unifying Theory of Dark Energy, Dark Matter, and Baryonic Matter in the Positive-Negative Mass Universe Pair: Protogalaxy and Galaxy Evolutions. Journal of Modern Physics, 11, 1091-1122.

https://doi.org/10.4236/jmp.2020.117069

Received: June 21, 2020

Accepted: July 20, 2020

Published: July 23, 2020

Copyright $\odot 2020$ by author(s) and Scientific Research Publishing Inc. This work is licensed under the Creative Commons Attribution International License (CC BY 4.0).

http://creativecommons.org/licenses/by/4.0/

(c) (i) Open Access

\begin{abstract}
This paper modifies the Farnes' unifying theory of dark energy and dark matter which are negative-mass, created continuously from the negative-mass universe in the positive-negative mass universe pair. The first modification explains that observed dark energy is $68.6 \%$, greater than $50 \%$ for the symmetrical positive-negative mass universe pair. This paper starts with the proposed positive-negative-mass 11D universe pair (without kinetic energy) which is transformed into the positive-negative mass $10 \mathrm{D}$ universe pair and the external dual gravities as in the Randall-Sundrum model, resulting in the four equal and separate universes consisting of the positive-mass $10 \mathrm{D}$ universe, the positive-mass massive external gravity, the negative-mass $10 \mathrm{D}$ universe and the negative-mass massive external gravity. The positive-mass 10D universe is transformed into $4 \mathrm{D}$ universe (home universe) with kinetic energy through the inflation and the Big Bang to create positive-mass dark matter which is five times of positive-mass baryonic matter. The other three universes without kinetic energy oscillate between 10D and 10D through 4D, resulting in the hidden universes when $\mathrm{D}>4$ and dark energy when $\mathrm{D}=4$, which is created continuously to our $4 \mathrm{D}$ home universe with the maximum dark energy $=3 / 4=75 \%$. In the second modification to explain dark matter in the $\mathrm{CMB}$, dark matter initially is not repulsive. The condensed baryonic gas at the critical surface density induces dark matter repulsive force to transform dark matter in the region into repulsive dark matter repulsing one another. The calculated percentages of dark energy, dark matter, and baryonic matter are 68.6 (as an input from the observation), 26 and 5.2, respectively, in agreement with observed 68.6, 26.5 and 4.9, respectively, and dark energy started in 4.33 billion years ago in agreement with the observed $4.71 \pm$
\end{abstract}


0.98 billion years ago. In conclusion, the modified Farnes' unifying theory reinterprets the Farnes' equations, and is a unifying theory of dark energy, dark matter, and baryonic matter in the positive-negative mass universe pair. The unifying theory explains protogalaxy and galaxy evolutions in agreement with the observations.

\section{Keywords}

Unifying Theory, Farnes, Dark Energy, Dark Matter, Baryonic Matter, Negative Mass, Positive-Negative Mass Universe Pair, Protogalaxy Evolution, Galaxy Evolution

\section{Introduction}

In the Farnes' unifying theory of dark energy and dark matter [1], dark energy and dark matter can be unified into a single negative-mass dark fluid which is created continuously from the negative-mass universe to our positive-mass universe. The model is a modified $\Lambda \mathrm{CDM}$ cosmology, and indicates that continuously-created negative masses can resemble the cosmological constant and can flatten the rotation curves of galaxies. In the first three-dimensional N-body simulations of negative mass dark matter in the scientific literature, dark matter naturally forms halos around galaxies that extend to several galactic radii. These halos are not cuspy, unlike the cuspy halos derived from the simulations of conventional positive-mass dark matter [2]. The core cusp is not observed [3]. Therefore, the Farnes' cosmological model is able to predict correctly the observed non-cuspy distribution of dark matter in galaxies from first principles.

This paper modifies and reinterprets the Farnes' unifying theory of dark energy and dark matter in the positive-negative mass universe pair model where positive-mass and negative-mass universes are symmetrical. (The Farnes' unifying theory does not assume symmetrical positive-negative mass universes.) The first modification is about dark energy. With the symmetry between positive-mass universe and negative-mass universe, the portion of dark energy to be created continuously from the negative-mass universe to our positive-mass universe cannot start from zero to more than $50 \%$. Since the observed portion of dark energy is $68.6 \%$ [4], for symmetrical positive-negative mass universe pair, dark energy is more than negative mass from the symmetrical negative-mass universe. As a result, the source of dark energy needs modification. Another modification is about dark matter. Evidence for early existence of dark matter comes from measurements on cosmological scales of anisotropies in the cosmic microwave background [5] [6] [7]. The CMB is the remnant radiation from the hot early days of the universe. The photons underwent oscillations that froze in just before decoupling from the baryonic matter at a redshift of 1100 . The angular scale and height of the peaks (and troughs) of these oscillations are powerful probes of cosmological parameters, including the total energy density, the ba- 
ryonic fraction, and the dark matter component. The CMB provides irrefutable evidence for the early existence of dark matter. The Farnes' unifying theory does not allow the early existence of positive-mass dark matter, so dark matter needs modification.

To deal with greater than $50 \%$ of dark energy and the early existence of dark matter, this paper proposes the positive-negative mass universe pair model where the positive and the negative universes are symmetrical [8] [9] [10]. This paper starts with the proposed positive-negative-mass 11D membrane-antimembrane universe pair (without kinetic energy) which are transformed into the positive-negative-mass 10D string-antistring universe pair and the external dual gravities as in the Randall-Sundrum model [11] [12], resulting in the four equal and separate universes consisting of the positive-mass 10D universe, the positive-mass massive external gravity, the negative-mass 10D universe, and the negative-mass massive external gravity. The positive-mass 10D universe is transformed into $4 \mathrm{D}$ universe (home universe) with kinetic energy through the inflation and the Big Bang. Dark matter is the right-handed neutrino, exactly five times of baryonic matter in total mass. The other three universes without kinetic energy oscillate between 10D and 10D through 4D, resulting in the hidden universes when $\mathrm{D}>4$ and dark energy when $\mathrm{D}=4$ (the maximum dark energy $=3 / 4=75 \%$ ). In this paper, the first modification is that $4 \mathrm{D}$ dark energy is created from the three oscillating dimensional universes (the positive-mass massive external gravity, the negative-mass universe, and the negative-mass massive external gravity) instead of only negative-mass universe in the Farnes' unifying theory. Otherwise, both the proposed dark energy and the Farnes' dark energy are the same.

To deal with the early existence of positive-mass dark matter in the CMB, the second modification is that the proposed dark matter in the CMB is same as the conventional positive-mass dark matter observed in the CMB. However, after the $\mathrm{CMB}$, the proposed positive-mass dark matter turned into repulsive positive-mass dark matter when the condensed baryonic gas reached the critical surface density (derived from the acceleration constant $a_{0}$ in MOND [13] [14]) which induces dark matter repulsive force to transform dark matter in the region into repulsive dark matter repulsing one another, corresponding to the Farnes' repulsive negative-mass dark matter. Therefore, the proposed dark matter and the Farnes' dark matter have different beginnings, but both end up with the same repulsive force. In this way, a galaxy actually started with a dark matter core-cusp without repulsive dark matter. The removal of the core cusp by repulsive dark matter afterward solves many difficult problems in protogalaxy and galaxy evolutions.

The theoretical calculated percentages of dark energy, dark matter, and baryonic matter are 68.6 (as an input from the observation), 26, and 5.2, respectively, in agreement with observed 68.6, 26.5, and 4.9 [15], respectively. According to the calculation, dark energy started in 4.33 billion years ago in agreement 
with the observed $4.71 \pm 0.98$ billion years ago [16]. In conclusion, the modified Farnes' unifying theory reinterprets the Farnes' equations, and is a unifying theory of dark energy, dark matter, and baryonic matter in the positive-negative mass universe pair. The unifying theory explains protogalaxy evolution and galaxy evolution in agreement with the observations.

In Section 2, unifying theory of dark energy and dark matter by Farnes is described. Section 3 explains the positive-negative mass universe pair. Section 4 describes protogalaxy and galaxy evolutions.

\section{Unifying Theory of Dark Energy and Dark Matter}

In the Farnes' unifying theory of dark energy and dark matter [1], dark energy and dark matter can be unified into a single negative-mass dark fluid which is created continuously through a creation tensor from the negative-mass universe to our positive-mass universe.

\subsection{The Creation Tensor for Dark Energy and Dark Matter}

In the Einstein's field equation,

$$
R_{\mu v}-\frac{1}{2} R g_{\mu v}+\Lambda g_{\mu v}=\frac{8 \pi G}{c^{4}} T_{\mu v}
$$

where $R_{\mu \nu}$ is the Ricci curvature tensor, $R$ is the scalar curvature, $g_{\mu \nu}$ is the metric tensor, $\Lambda$ is the cosmological constant, $G$ is Newton's gravitational constant, $c$ is the speed of light in vacuum, and $T_{\mu \nu}$ is the stress-energy tensor.

In a homogeneous and isotopic universe, the Friedmann equation is

$$
H^{2} \equiv\left(\frac{\dot{a}}{a}\right)^{2}=\frac{8 \pi G}{3} \rho+\frac{\Lambda c^{2}}{3}-\frac{k c^{2}}{a^{2}}
$$

and the Friedmann acceleration equation.

$$
\left(\frac{\ddot{a}}{a}\right)=-\frac{4 \pi G}{3}\left(\rho+\frac{3 p}{c^{2}}\right)+\frac{\Lambda c^{2}}{3}
$$

where $a$ is the scale factor, $H$ is the Hubble parameter, $\rho$ is the total mass density of the universe, $p$ is the pressure, $k$ is the curvature parameter or intrinsic curvature of space, and $k / a^{2}$ is the spatial curvature in any time-slice of the universe. $k=+1,0$, and -1 , indicate a closed, flat, and open universe respectively. In the positive mass universe corresponding to the standard matter-dominated universe solutions with a critical density given by $\rho_{c}=3 H^{2} / 8 \pi G$ and a total density parameter given by $\Omega=\rho / \rho_{\mathcal{c}}$ where $\Omega=1,<1$, and $>1$, correspond to critical density, open, and closed universes respectively.

In the Farnes' unifying theory of dark energy and dark matter [1], baryonic matter has positive mass, while dark fluid consisting of dark energy and dark matter has negative masses. Dark fluid is created continuously through a "creation tensor" from the positive-mass dominated universe. Negative mass is created through the creation tensor, $C_{\mu v}$

$$
T_{\mu v}^{\prime}=T_{\mu v}+C_{\mu v}
$$


Einstein's field equations are therefore modified to

$$
R_{\mu v}-\frac{1}{2} R g_{\mu v}+\Lambda g_{\mu v}=\frac{8 \pi G}{c^{4}}\left(T_{\mu v}+C_{\mu v}\right)
$$

The creation tensor generates negative mass density, $\rho_{-}$. Farnes proposed that the Friedmann equation can be written in terms of both the cosmological constant and negative mass density, $\rho_{-}$as follows.

$$
\begin{aligned}
\left(\frac{\dot{a}}{a}\right)^{2} & =\frac{8 \pi G}{3} \rho_{+}+\frac{\Lambda c^{2}}{3}-\frac{k c^{2}}{a^{2}} \\
& =\frac{8 \pi G}{3} \rho_{+}+\frac{8 \pi G}{3} \rho_{-}-\frac{k c^{2}}{a^{2}}
\end{aligned}
$$

In this way, the cosmological constant $(\Lambda)$ is equivalent to the negative-mass density, and $\Lambda=8 \pi G \rho_{-} / c^{2}$. The accelerated cosmic expansion is caused by negative mass which is created continuously through the creation tensor. As these negative masses can take the form of a cosmological constant, the field equations are modified to

$$
R_{\mu v}-\frac{1}{2} R g_{\mu v}+\Lambda g_{\mu v}=\frac{8 \pi G}{c^{4}}\left(T_{\mu v}^{+}+T_{\mu v}^{-}+C_{\mu v}\right)
$$

In the Farnes' unifying theory for dark matter, the positive-mass baryonic galaxy is surrounded by a halo of continuously-created negative masses, with constant density $\rho_{-}$and of total mass $M_{-}$. The positive mass particle is now immersed in a negative mass fluid that behaves with resemblance to a cosmological constant with $\Lambda=8 \pi G \rho_{-} / c^{2}$.

Negative-mass dark matter repulses each other, while dark matter still attracts to baryonic matter. The solution for the rotation curve with circular velocity, $v$, is deduced as follows.

$$
v=\sqrt{\frac{G M_{*}}{r}-\frac{\Lambda c^{2}}{3} r^{2}}=\sqrt{\frac{G M_{*}}{r}-\frac{8 \pi G \rho_{-}}{3} r^{2}}
$$

where $r$ is the distance from a central point mass $M_{\star}$ and $\rho_{-}$is the local negative mass density. For $\Lambda=0$ or $\rho_{-}=0$, the standard Keplerian rotation curve is obtained. However, for non-zero values of the cosmological constant or $\rho_{-}$, the rotation curve is modified. A negative cosmological constant flattens the rotation curve, causing a steady increase at larger galactic radii in agreement with the observed rotation curve.

In a $\mathrm{N}$-body simulations of galaxy formation via negative-mass dark matter and positive-mass baryonic matter, Farnes starts with the galaxy that comprises 5000 particles, with a total positive mass of $M_{+}=1.0$. The initial positive mass particle distribution is located at the center of a cube of negative masses with volume $200^{3}$. The initial conditions of these negative masses are set to be uniformly distributed in position and with zero initial velocity. The negative mass sea comprises 45,000 particles, with a total mass of $M_{-}=-3.0$. The negative masses at the outskirts of the cube are mutually-repelled by other surrounding negative masses and the cube begins to expand in volume. Meanwhile, the nega- 
tive masses within the central portion of the cube are attracted towards the positive mass galaxy. From their initially zero velocities, the negative mass particles are slushed to-and-fro from either side of the positive mass galaxy. Eventually, the negative mass particles reach dynamic equilibrium in a halo that surrounds the positive mass galaxy and which extends out to several galactic radii. The negative mass particles have naturally formed a dark matter halo to have a flat central dark matter distribution with the constant dark matter density core.

With positive mass dark matter, the Navarro-Frenk-White (NFW) profile [2] derived from standard N-body simulations has the high density dark matter at the core of galaxy in disagreement with the observed distribution of dark matter. The high density dark matter at the core of galaxy is not observed [3]. This is known as the core-cusp problem or the cuspy halo problem and is currently unsolved [17]. A negative mass cosmology can therefore provide a solution to the cuspy halo problem by the repulsion within dark matter particles. This appears to be the only cosmological theory within the scientific literature that can explain and predict correctly the non-cuspy distribution of dark matter in galaxies from first principles of intrinsic repulsive dark matter. Dark matter of low-mass sterile neutrino and polytropic exponent under fermion degeneracy pressure also shows non-cuspy distribution [18].

\subsection{The Modified Creation Tensor for Dark Energy and Dark Matter}

This paper modifies and reinterprets the Farnes' unifying theory of dark energy and dark matter as negative-mass dark fluid derived from the positive-negative mass universe pair model where positive-mass and negative-mass universes are symmetrical. (The Farnes' unifying theory does not assume symmetrical positive-negative mass universes.) The first modification is about dark energy. With the symmetry between positive mass and negative mass, the portion of dark energy created through the creation tensor cannot start from zero to more than $50 \%$. Since dark energy is $68.6 \%$, dark energy is more than negative mass, and the creation tensor for dark energy needs modification. The first modification is to modify the origin of dark energy. The second modification is to modify dark matter. Evidence for early existence of dark matter comes from measurements on cosmological scales of anisotropies in the cosmic microwave background [5] [6] [7]. The CMB is the remnant radiation from the hot early days of the universe. The photons underwent oscillations that froze in just before decoupling from the baryonic matter at a redshift of 1100 . The angular scale and height of the peaks (and troughs) of these oscillations are powerful probes of cosmological parameters, including the total energy density, the baryonic fraction, and the dark matter component. The CMB provides irrefutable evidence for the early existence of dark matter. The Farnes' unifying theory does not allow the early existence of positive dark matter, so the creation tensor for dark matter needs modification. The second modification is to modify the origin of dark matter. 


\section{The Positive-Negative Mass Universe Pair Model}

To deal with more than $50 \%$ of dark energy and the early existence of dark matter in the CMB, this paper proposes as described previously [8] [9] [10] the positive-negative mass universe pair model where the positive and the negative universes are symmetrical. The positive-negative mass universe pair model involves the oscillating spacetime dimension number, the space structure, and the cyclic universe model.

\subsection{The Oscillating Spacetime Dimension Number}

The oscillating spacetime dimension number oscillates between 11D membrane and 11D membrane through 10D string and between 10D particle and 10D particle through $4 \mathrm{D}$ particle as described in the previous papers [19] [20]. Because membrane and string require $11 \mathrm{D}$ and $10 \mathrm{D}$, respectively, any spacetime dimension below 10D has to be the spacetime dimension for particle which allows any spacetime dimension numbers.

\subsubsection{The Membrane-String 0scillation between 11D4d and 11D4d} In the membrane-string oscillation, the $11 \mathrm{D}$ brane is transformed into the $10 \mathrm{D}$ string with an extra dimension. This 11D warped brane world in the 10D string with compact extra dimension is analogous to the 5D warped brane world in our $4 \mathrm{D}$ universe with compact extra dimension in the Randall-Sundrum model [11] [12]. The RS1 of the Randall-Sundrum model produces the two different branes consisting of the Tevbrane and the Planckbrane. The Planckbrane has very strong gravity, while the Tevbrane has all other forces and extremely weak gravity. There are boundaries between the Tevbrane and the Planckbrane. The Tevbrane corresponds to our universe with extremely weak gravity comparing with other forces. Almost all gravity is external gravity outside of our universe. The Randall-Sundrum model explains the hierarchy problem between gravity and other forces.

In the same way as the RS1 in the Randall-Sundrum model, two 11D membranes produce the $10 \mathrm{D}$ string corresponding to the Tevbrane and the external gravity corresponding to the Planckbrane. The 11D membrane, the 10D string, and the external gravity have about the same energy. The 10D string has the extremely weak internal gravity as in the Tevbrane with an extremely weak gravity in the Randall-Sumdrum model.

The RS1 in the Randall-Sundrum model

$5 \mathrm{D}$ brane world $\stackrel{\text { compact extra space dimension }}{\longrightarrow}$ Tevbrane + Planckbrane

two 11D membranes $\stackrel{\text { oscillation between 11D membrane and 10D string }}{\longleftrightarrow} 10 \mathrm{D}$ string + external gravity

The membrane-string oscillation is reversible, so the $10 \mathrm{D}$ string and the external gravity can also reverse back to the $11 \mathrm{D}$ membrane.

\subsubsection{The Particle Oscillation between 10D and 10D through 4D}

As described previously [8] [9] [10], the space-time dimension numbers oscillate 
reversibly between $10 \mathrm{D}$ and $4 \mathrm{D}$ reversibly dimension by dimension without compactization. The oscillating space-time numbers from 10D to $4 \mathrm{D}$ relate to varying speed of light. Varying speed of light has been proposed to explain the horizon problem of cosmology [21] [22]. J. D. Barrow [23] proposes that the time dependent speed of light varies as some power of the expansion scale factor $a$ in such way that

$$
c(t)=c_{0} a^{n},
$$

where $c$ is the speed of light and $n$ are constants. The increase of speed of light is continuous.

In this paper, the speed of light is invariant in a constant space-time dimension number, and the speed of light varies with varying space-time dimension number from 4 to 10 as follows.

$$
c_{\mathrm{D}}=c / \alpha^{\mathrm{D}-4},
$$

where $c$ is the observed speed of light in the $4 \mathrm{D}$ space-time, $c_{\mathrm{D}}$ is the quantized varying speed of light in space-time dimension number, $\mathrm{D}$, from 4 to 10 , and $\alpha$ is the fine structure constant for electromagnetism. The speed of light increases with the increasing space-time dimension number $D$. Since the speed of light for $>4 \mathrm{D}$ particle is greater than the speed of light for $4 \mathrm{D}$ particle, the observation of $>4 \mathrm{D}$ particles by $4 \mathrm{D}$ particles violates casualty. Thus, $>4 \mathrm{D}$ particles are hidden particles with respect to $4 \mathrm{D}$ particles. Particles with different space-time dimensions are transparent and oblivious to one another, and separate from one another if possible.

As described previously [8] [9] [10], the particle oscillation between 10D and 10D through $4 \mathrm{D}$ involves mass dimension (denoted as $\mathrm{d}$ ) to represent the mass. In the initial condition for the oscillation, $\mathrm{D}+\mathrm{d}=14$ where $\mathrm{D}$ and $\mathrm{d}$ are between 4 and 10. For an example, a dimension has a dual spacetime-mass dimension numbers of $10 \mathrm{D} 4 \mathrm{~d}$ or $4 \mathrm{D} 10 \mathrm{~d}$. The transformations for oscillating dimension number between 10D and 4D consist of the varying speed of light dimensional (VSLD) transformation for spacetime dimension $\mathrm{D}$ and the varying supersymmetry dimensional (VSD) transformation for mass dimension d. For the VSLD transformation for D, $E=M_{0} c^{2}$ modified by Equation (12) is expressed as

$$
\begin{gathered}
E=M_{0} c_{\mathrm{D}}^{2}=M_{0} \cdot\left(c^{2} / \alpha^{2(\mathrm{D}-4)}\right), \\
M_{0, \mathrm{D}, \mathrm{d}}=M_{0, \mathrm{D}-n, \mathrm{~d}+n} \alpha^{2 n}, \\
E_{\text {vacuum }, \mathrm{D}}=E-M_{0, \mathrm{D}} c^{2}, \\
\mathrm{D}, \mathrm{d} \stackrel{\text { vsLD transformation }}{\longrightarrow}(\mathrm{D} \mp n),(\mathrm{d} \pm n)
\end{gathered}
$$

where $c_{\mathrm{D}}$ is the quantized varying speed of light in space-time dimension number, $D$, from 4 to $10, c$ is the observed speed of light in the $4 \mathrm{D}$ space-time, $\alpha$ is the fine structure constant for electromagnetism, $E$ is energy, $M_{0}$ is rest mass, $\mathrm{D}$ is the space-time dimension number from 4 to $10, \mathrm{~d}$ is the mass dimension number from 4 to $10, n$ is an integer, and $E_{\text {vacuum }}=$ vacuum energy. From Equa- 
tion (12), $10 \mathrm{D}$ has the lowest rest mass, and $4 \mathrm{D}$ has the highest rest mass. According to the calculation from Equation (13), the rest mass of $4 \mathrm{D}$ is $1 / \alpha^{12} \approx$ $137^{12}$ times of the mass of 10D. From Equation (14), 10D has the highest vacuum energy, while $4 \mathrm{D}$ particle has zero vacuum energy. A particle with $10 \mathrm{D}$ is transformed to a particle with 4D from Equation (15) through the VSLD transformation. Spacetime dimension number decreases with decreasing speed of light, decreasing vacuum energy, and increasing rest mass. The $4 \mathrm{D}$ and the $10 \mathrm{D}$ have zero and the highest vacuum energies, respectively.

In the normal supersymmetry transformation, the repeated application of the fermion-boson supersymmetry transformation carries over a boson (or fermion) from one point to the same boson (or fermion) at another point at the same mass, resulting in translation without changing mass. Under the varying supersymmetry dimensional (VSD) transformation, the repeated application of the fermion-boson supersymmetry transformation carries over a boson from one point to the boson at another point at different mass dimension number at different mass, resulting in translation and fractionalization or condensation. The repeated VSD transformation carries over a boson $B_{d}$ into a fermion $F_{d}$ and a fermion $\mathrm{F}_{\mathrm{d}}$ to a boson $\mathrm{B}_{\mathrm{d}-1}$, which can be expressed as follows.

$$
\begin{gathered}
M_{\mathrm{d}, \mathrm{F}}=M_{\mathrm{d}, \mathrm{B}} \alpha_{\mathrm{d}, \mathrm{B}}, \\
M_{\mathrm{d}-1, \mathrm{~B}}=M_{\mathrm{d}, \mathrm{F}} \alpha_{\mathrm{d}, \mathrm{F}},
\end{gathered}
$$

where $M_{\mathrm{d}, \mathrm{B}}$ and $M_{\mathrm{d}, \mathrm{F}}$ are the masses for a boson and a fermion, respectively, $\mathrm{d}$ is the mass dimension number, and $\alpha_{\mathrm{d}, \mathrm{B}}$ or $\alpha_{\mathrm{d}, \mathrm{F}}$ is the fine structure constant that is the ratio between the masses of a boson and its fermionic partner. where $M_{\mathrm{d}, \mathrm{B}}$ and $M_{\mathrm{d}, \mathrm{F}}$ are the masses for a boson and a fermion, respectively, $\mathrm{d}$ is the mass dimension number, and $\alpha_{\mathrm{d}, \mathrm{B}}$ or $\alpha_{\mathrm{d}, \mathrm{F}}$ is the fine structure constant that is the ratio between the masses of a boson and its fermionic partner. Assuming $\alpha$ 's are the same, it can be expressed as

$$
M_{\mathrm{d}, \mathrm{B}}=M_{\mathrm{d}+1, \mathrm{~B}} \alpha_{\mathrm{d}+1}^{2} .
$$

The oscillating dimension number transformation between 10D4d and 10D4d through 4D4d involves both the VSLD transformation and the VSD transformation as the stepwise two-step transformation as follows. stepwise two-step varying transformation

$$
\begin{aligned}
& \text { (1) } \mathrm{D}, \mathrm{d} \stackrel{\mathrm{VSLD}}{\longleftrightarrow}(\mathrm{D} \mp 1),(\mathrm{d} \pm 1) \\
& \text { (2) } \mathrm{D}, \mathrm{d} \stackrel{\mathrm{VSD}}{\longleftrightarrow} \mathrm{D},(\mathrm{d} \pm 1)
\end{aligned}
$$

The repetitive stepwise two-step dimension number oscillation between 10D4d and 10D4d through 4D4d as follows.

$$
\begin{aligned}
& 10 \mathrm{D} 4 \mathrm{~d} \rightarrow \text { 9D5d } \rightarrow \text { 9D4d } \rightarrow \text { 8D5d } \rightarrow \text { 8D4d } \rightarrow \text { 7D5d } \rightarrow \text { 7D4d } \rightarrow \text { 6D5d } \\
& \rightarrow \text { 6D4d } \rightarrow \text { 5D5d } \rightarrow \text { 5D4d } \rightarrow \text { 4D5d } \rightarrow \text { 4D4d } \rightarrow \text { 5D4d } \rightarrow \text { 5D5d } \rightarrow \text { 6D4d } \\
& \rightarrow \text { 6D5d } \rightarrow \text { 7D4d } \rightarrow \text { 7D5d } \rightarrow \text { 8D4d } \rightarrow \text { 8D5d } \rightarrow \text { 9D4d } \rightarrow \text { 9D5d } \rightarrow 10 \text { D4d }
\end{aligned}
$$

From Equation $(18)$, the mass of $9 \mathrm{D} 4 \mathrm{~d}$ is $\alpha^{2} \approx(1 / 137)^{2}$ times of the mass of 9D5d through the varying supersymmetry transformation. The transformation 
from a higher mass dimensional particle to the adjacent lower mass dimensional particle is the fractionalization of the higher dimensional particle to the many lower dimensional particles in such way that the number of lower dimensional particles becomes

$$
N_{\mathrm{d}-1}=N_{\mathrm{d}} / \alpha^{2} \approx N_{\mathrm{d}}(137)^{2}
$$

The fractionalization also applies to D for 10D4d to 9D4d, so

$$
N_{\mathrm{D}-1}=N_{\mathrm{D}} / \alpha^{2}
$$

Since the supersymmetry transformation involves translation, this stepwise varying supersymmetry transformation leads to a translational fractionalization, resulting in the cosmic expansion. Afterward, the QVSL transformation transforms 9D4d into 8D5d with a higher mass. The two-step transformation repeats until 4D4d, and then reverses stepwise back to $10 \mathrm{D} 4 \mathrm{~d}$ for the cosmic contraction. The oscillation between $10 \mathrm{D}$ and $4 \mathrm{D}$ results in the reversible cyclic fractionalization-contraction for the reversible cyclic expansion-contraction of the universe which does not involve irreversible kinetic energy.

\subsection{The Space Structure}

In the space structure, attachment space that attaches matter to the space relates to rest mass, and detachment space that detaches matter from the space relates to kinetic energy. Attachment space is the space precursor of the transitional Higgs field, and detachment space is the space precursor of the transitional reverse Higgs field.

\subsubsection{The Higgs Mechanism}

In conventional physics, space does not couple with particles, and is the passive zero-energy ground state space. Under spontaneous symmetry breaking in conventional physics, the passive zero-energy ground state is converted into the active, permanent, and ubiquitous nonzero-energy Higgs field, which couples with massless particle to produce the transitional Higgs field-particle composite. Under spontaneous symmetry restoring, the transitional Higgs field-particle composite is converted into the massive particle with the longitudinal component on zero-energy ground state without the Higgs field as follows.

$$
\begin{aligned}
& \text { zero-energy groud state space } \stackrel{\text { spontaneous symmetry breaking }}{\longrightarrow} \\
& \text { nonzero-energy scalar Higgs field } \stackrel{\text { massless particle }}{\longrightarrow} \\
& \stackrel{\text { the transitional nonzero-energy Higgs field-particle composite] }}{\stackrel{\text { spontaneous symmetry restoring }}{\longrightarrow} \text { massive particle with the longitudinal component }}
\end{aligned}
$$

on zero-energy ground state space without the Higgs field

In conventional physics, the nonzero-energy scalar Higgs Field exists permanently in the universe. The problem with such nonzero-energy field is the cosmological constant problem from the huge gravitational effect by the nonzero-energy Higgs field in contrast to the observation [24]. 
Unlike passive space in conventional physics, attachment space actively couples to massless particle. Under spontaneous symmetry breaking, attachment space as the active zero-energy ground state space couples with massless particle to form momentarily the transitional non-zero energy Higgs field-particle composite. The Higgs field is momentary and transitional, avoiding the cosmological constant problem. Under spontaneous symmetry restoring, the transitional nonzero-energy Higgs field-particle composite is converted into massive particle with the longitudinal component on zero-energy attachment space without the Higgs field as follows.

massless particle + zero-energy attachment space $\stackrel{\text { spontaneous symmetry breaking }}{\longrightarrow}$

[the transitional non-zero energy Higgs field-particle composite]

$\stackrel{\text { spontaneous symmetry restoring }}{\longrightarrow}$ massive particle with the longitudinal component

on zero-energy attachment space without the Higgs field

Detachment space is the space precursor of the reverse Higgs field. Unlike the conventional model, detachment space actively couples to massive particle. Under spontaneous symmetry breaking, the coupling of massive particle to zero-energy detachment space produces the transitional nonzero-energy reverse Higgs field-particle composite which under spontaneous symmetry restoring produces massless particle on zero-energy detachment space without the longitudinal component without the reverse Higgs field as follows.

massive particle + zero-energy detachment space $\stackrel{\text { spontaneous symmetry breaking }}{\longrightarrow}$

[the transitional nonzero-energy reverse Higgs field-particle composite]

$\stackrel{\text { spontaneous symmetry restoring }}{\longrightarrow}$ massless particle without the longitudinal component

on zero-energy detachment space without the reverse Higgs field

For the electroweak interaction in the Standard model where the electromagnetic interaction and the weak interaction are combined into one symmetry group, under spontaneous symmetry breaking, the coupling of the massless weak W, weak Z, and electromagnetic A (photon) bosons to zero-energy attachment space produces the transitional nonzero-energy Higgs fields-bosons composites which under partial spontaneous symmetry restoring produce massive $\mathrm{W}$ and $\mathrm{Z}$ bosons on zero-energy attachment space with the longitudinal component without the Higgs field, massless A (photon), and massive Higgs boson as follows.

massless $\mathrm{WZ}+$ zero-energy $\mathrm{WZ}$ attachment space + massless A

+ zero-energy A attachment space $\mathrm{A} \stackrel{\text { spontaneous symmetry breaking }}{\longrightarrow}$

[the transitional nonzero-energy WZ Higgs field - WZ composite]

+ [nonzero-energy A Higgs field - A composite] $\stackrel{\text { partial spontaneous symmetry restoring }}{\longrightarrow}$

massive $\mathrm{WZ}$ with the longitudinal component on attachment space without

the Higgs field + massless A + the nonzero energy massive Higgs boson

In terms of mathematical expression, the conventional permanent Higgs field model and the transitional Higgs field model are identical. The interpretations of 
the mathematical expression are different for the permanent Higgs field model and the transitional Higgs field model. The transitional Higgs field model avoids the cosmological problem in the permanent Higgs field model.

In the Higgs mechanism, gauge bosons are assumed to be massless originally. Elementary fermions (leptons and quarks) can be assumed to be massive originally. However, the observed neutrinos are nearly massless and left-handed only. The paper posits that the left handed became massless through the reverse Higgs mechanism. For the symmetrical massive left handed neutrinos and right-handed neutrinos under spontaneous symmetry breaking, the coupling of the massive left handed neutrinos and the massive right handed neutrinos to zero-energy detachment space produces the transitional nonzero-energy reverse Higgs fields-neutrinos composites which under partial spontaneous symmetry restoring produce massless left handed neutrinos on zero-energy detachment space without the longitudinal component without the reverse Higgs field, massive right-handed neutrinos (dark matter), and the massive reverse Higgs boson as follows.

$$
\begin{aligned}
& \text { massive } v_{L}+\text { zero energy } v_{L} \text { detachment space }+ \text { massive } v_{R} \\
& + \text { zero-energy } v_{R} \text { detachment space } \stackrel{\text { spontaneous symmetry breaking }}{\longrightarrow} \\
& \text { [the transitional nonzero-energy } v_{L} \text { reverse Higgs field }-v_{L} \text { composite] } \\
& +\left[\text { nonzero-energy } v_{R} \text { reverse Higgs field }-v_{R}\right. \text { composite] } \\
& \stackrel{\text { partial spontaneous symmetry restoring }}{\longrightarrow} \text { massless } v_{L} \text { without the longitudinal } \\
& \text { component on detachment space without the Higgs field } \\
& \text { +massive } v_{R}+\text { the nonzero energy massive reverse Higgs boson }
\end{aligned}
$$

As described in the previous paper [25], the reverse Higgs boson was observed as the two unusual steeply upward-going ultra-high-energy (UHE) cosmic ray events with energies of $\approx 0.6 \mathrm{EeV}[26]$ and $\approx 0.56 \mathrm{EeV}$ [27] in the Antarctic Impulsive Transient Antenna (ANITA) experiment [28]. These shower events have the characteristics of the decay of a tau lepton, which emerges from the surface of the ice, and the tau lepton is explained as the product of a UHE parent tau neutrino by the charged-current interactions with the Earth matter. However, such UHE tau neutrino cannot survive the passage through the Earth. The previous paper posits that the upward-going ANITA events are derived from the cosmic ray of the baryonic-dark matter reverse Higgs boson that travels through the Earth. The calculated value for the reverse Higgs boson is $0.47 \mathrm{EeV}$ in good agreement with the observed 0.56 and $0.6 \mathrm{EeV}$. As shown in Section 3.3., dark matter (sterile neutrinos) is part of the periodic table of elementary particles for baryonic matter and dark matter.

\subsubsection{The Three Spaces}

The combination of $n$ units of attachment space as 1 and $n$ units of detachment space as 0 brings about three different spaces: binary partition space, miscible space, or binary lattice space as below.
$(1)_{n}$
$(0)_{n}$
$(1)_{n}(0)_{n}$,
$(1+0)_{n} \quad$ or
$\left(\begin{array}{ll}1 & 0\end{array}\right)_{n}$

attachment space detachment space binary partition space, miscible space, binary lattice space 
Binary partition space, $(1)_{n}(0)_{n}$, consists of two separated continuous phases of multiple quantized units of attachment space and detachment space, and it is the space structure for wave-particle duality in quantum mechanics. In miscible space, $(1+0)_{n}$, attachment space is miscible to detachment space, and there is no separation of attachment space and detachment space, and it is the space structure for miscible mass-energy in relativity. Binary lattice space, $\left(\begin{array}{ll}1 & 0\end{array}\right)_{n}$, consists of repetitive units of alternative attachment space and detachment space, and it is the space structure for virtual particles in quantum field theory.

An object in binary partition space $(1)_{n}(0)_{n}$ has both movement and rest at the same time, resulting in wave-particle duality for movement-rest duality in quantum mechanics. An object in binary partition space cannot be completely at movement (zero momentum) or completely at rest (zero distance), resulting in the uncertainty principle as follows.

$$
\sigma_{x} \sigma_{p} \geq \frac{\hbar}{2}
$$

where $x$ is position and $p$ is momentum. The interference to binary partition space collapses binary partition space, resulting in miscible space as follows.

$$
\begin{aligned}
& (0)_{n}(1)_{n} \stackrel{\text { collapse }}{\longrightarrow}(0+1)_{n} \\
& \text { binary partition space miscible space }
\end{aligned}
$$

In miscible space, attachment space is miscible to detachment space, resulting in miscible mass and energy where attachment space for mass provides zero speed for rest mass $m_{0}$, while detachment space for energy provides the speed of light for kinetic energy. The total energy is the combination of both as follows.

$$
E=K+m_{0} c^{2}=\gamma m_{0} c^{2}
$$

where $\gamma=1 /\left(1-v^{2} / c^{2}\right)^{1 / 2}$ is the Lorentz factor for time dilation, $m_{0}$ is rest mass,

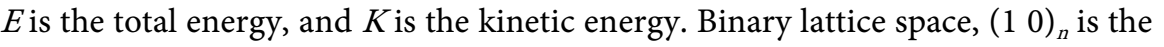
space structure for virtual particles in quantum field theory which will be described in the next section.

\subsection{The Cyclic Universes Model}

The seven steps in the cyclic universes model are 1) the formation of positive-mass and negative-mass dual 11D4d membrane-antimembrane universes from the zero-mass inter-universal void, 2) the transformation of the $11 \mathrm{D} 4 \mathrm{~d}$ membrane-antimembrane dual universes to the 10D4d string-antistring dual universes and dual external dual gravities, 3) the transformation from the string-antistring dual universes to the particle-antiparticle dual universes, 4) the transformation of the positive-mass $10 \mathrm{D} 4 \mathrm{~d}$ universe into the positive-mass $4 \mathrm{D}$ universe, and the transformation of the other three universes into the hidden oscillating dimension number universes from $10 \mathrm{D}$ to $5 \mathrm{D}, 5)$ the transformation of all four universes into the $4 \mathrm{D}$ universes, 6) the positive-mass $4 \mathrm{D}$ universe and the three hidden oscillating dimension number from $5 \mathrm{D}$ to $10 \mathrm{D}$, and 7 ) the return to the 10D4d particle-antiparticle universes (the step 3) as in Figure 1 and Figure 2. 


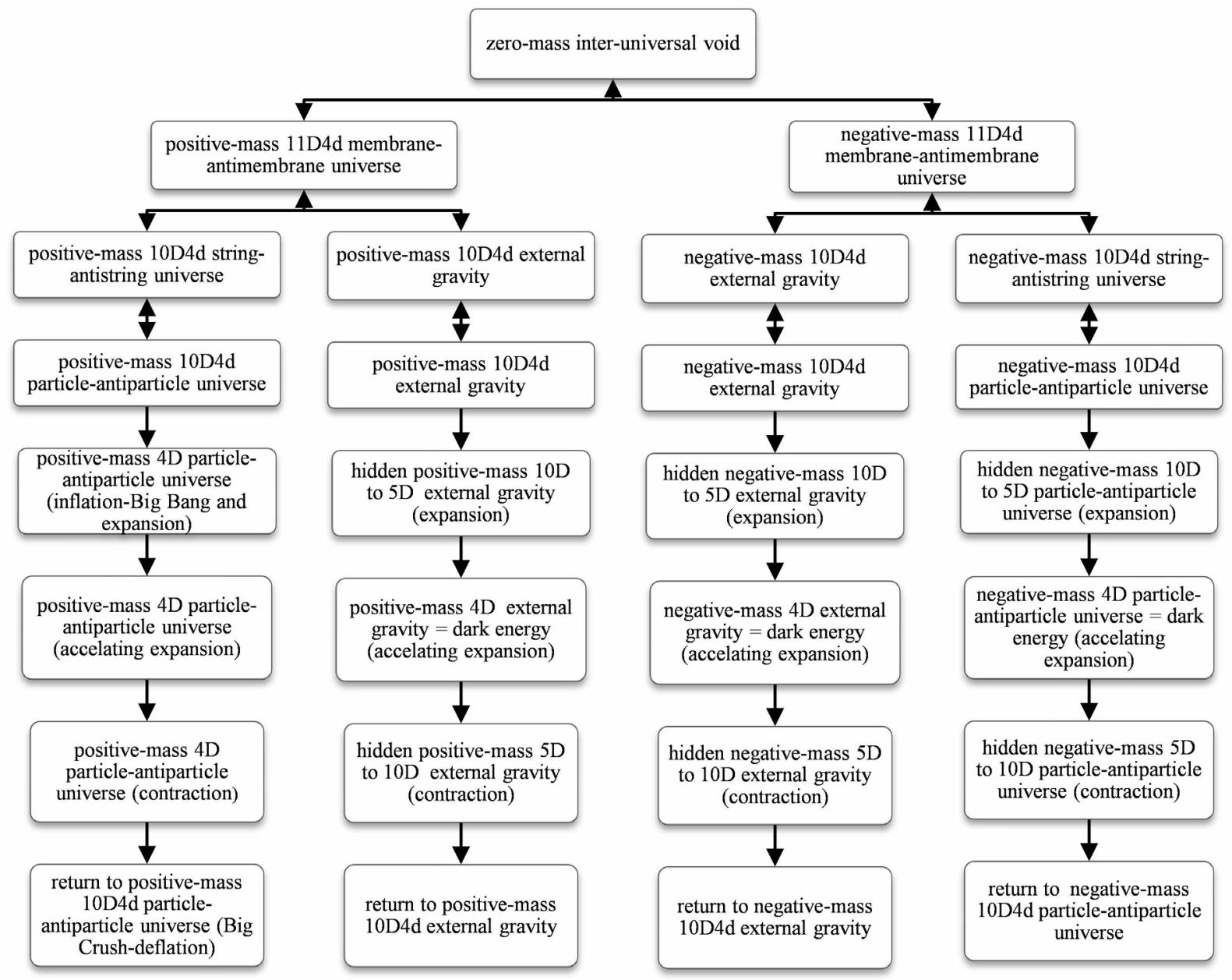

Figure 1. The cyclic universes model.

zero-mass Inter-universal void

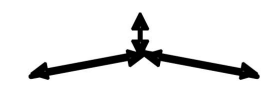

+ 11D4d membrane-antimembrane universe $\quad-11 \mathrm{D} 4 \mathrm{~d}$ membrane-antimembrane universe

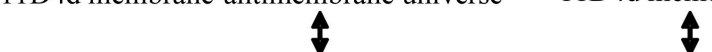

+10D4d string-antistring universe- gravity $\quad-10 \mathrm{D} 4 \mathrm{~d}$ string-antistring universe- gravity 4

+10D4d particle-antiparticle universe-gravity $\quad-10 \mathrm{D} 4 \mathrm{~d}$ particle-antiparticle universe-gravity

$\begin{array}{llll}\text { +4D particle- } & - \text { hidden 10D } & \text { +4D particle- } & - \text { hidden 5D to } \\ \text { antiparticle } & \text { to 5D particle- } & \text { antiparticle } & \text { 10D particle- } \\ \text { universe-gravity } & \text { antiparticle } & \text { universe-gravity } & \text { antiparticle } \\ \text { (inflation-Big } & \text { universe-gravity } & \text { (contraction-Big } & \text { universe-gravity } \\ \text { Bang- } & \text { (expansion) } & \text { Crush-deflation) } & \text { (contraction) }\end{array}$

expansion)

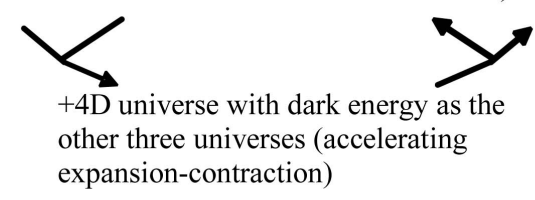

$+=$ positive-mass, $-=$ negative-mass, gravity $=$ external gravity

Figure 2. The cyclic universes model. 
1) the formation of the positive-negative-mass 11D4d membrane-antimembrane dual universes

In the cyclic universes model, the universes start with the positive-mass 11D4d membrane-antimembrane universe and the negative-mass $11 \mathrm{D} 4 \mathrm{~d}$ membrane-antimembrane universe derived from the zero-mass inter-universal void. The mass sum of the dual universes is zero. The zero-mass inter-universal void and the dual universe are reversible, so the dual universe can reverse back to the zero-mass inter-universal void. The inter-universal void contains only detachment space to prevent irreversible inter-universal collision, while the dual universes contain only attachment space without kinetic energy. These 11D dual universes are the universes with the oscillating spacetime dimension number, and start the process of oscillation between 11D and 11D through 10D and between 10D and 10D through 4D.

2) the transformation of the 11D4d membrane-antimembrane dual universes to the 10D4d string-antistring dual universes and dual external gravities

As described in Section 3.1.1., the transformation of the 11D membrane produces the $10 \mathrm{D}$ string and the external gravity. The results are the positive-mass $10 \mathrm{D} 4 \mathrm{~d}$ string-antistring universe, the positive-mass external gravity, the negative-mass 10D4d string-antistring universe, and the negative-mass external gravity. These four universes are separate, and have equal energy. The 10D4d string-external gravity and the 11D4d membrane are reversible.

3) the transformation from the string-antistring dual universes to the particle-antiparticle dual universes

Since string exists only in $10 \mathrm{D}$, so any further transformation of $\mathrm{D}$ to lower than 10 cannot be string. As a result, to transform lower than 10, string-antistring is converted into particle-antiparticle. The results are the positive-mass 10D4d particle-antiparticle universe, the positive-mass 10D4d external gravity, the negative-mass $10 \mathrm{D} 4 \mathrm{~d}$ particle-antiparticle universe, and the negative-mass $10 \mathrm{D} 4 \mathrm{~d}$ external gravity. Particle-antiparticle and string-antistring are reversible.

4) the transformation of the positive-mass 10D4d particle-antiparticle universe into the positive-mass $4 \mathrm{D}$ universe, and the transformation of other three universes into the hidden oscillating dimension number universes from 10D to $\underline{5 \mathrm{D}}$

The positive-mass 10D particle-antiparticle universe is transformed into the positive-mass $4 \mathrm{D}$ universe to produce the $4 \mathrm{D}$ standard model particles. Under the oscillating spacetime dimension number, the negative-mass 10D particle-antiparticle, the positive-mass external gravity, and the negative-mass external gravity are transformed into the hidden oscillating dimension number universes from $10 \mathrm{D}$ to $5 \mathrm{D}$.

4a) the formation of the positive-mass $4 \mathrm{D}$ particle-antiparticle universe

The formation of the positive-mass $4 \mathrm{D}$ particle-antiparticle universe includes the inflation and followed by the Big Bang. The inflation involves the VSLD transformation from 10D4d to $4 \mathrm{D} 10 \mathrm{~d}$, because from Equation (13), the rest mass 
$M_{0}$ of $4 \mathrm{D} 10 \mathrm{~d}$ is $M_{0,10}=M_{0,4} / \alpha^{2(10-4)} \approx 137^{12}$ times of the rest mass of $10 \mathrm{D} 4 \mathrm{~d}$, resulting in the inflation for the rapid expansion

The Big Bang involves the entrance of detachment space from the inter-universal void to the positive-mass universe. Detachment space introduces massless particles and kinetic energy for the cosmic expansion, and forms the three spaces with attachment space. The Big Bang consists of the two steps. In the first step, all particles are converted into massless particles by detachment space for the reverse Higgs field as in Equation (25). The second step involves the partial conversion of massless particles into massive particles by attachment space for the Higgs field to produce massless particles, massive particles, and the Higgs boson as in Equations (26) and (27) for the standard model. The emergence of detachment space starts kinetic energy which causes the cosmic expansion as the Big Bang.

the inflation and the Big Bang (detachment space + partial attachment space)

$10 \mathrm{D} 4 \mathrm{~d} \stackrel{\text { the inflation }}{\longrightarrow} 4 \mathrm{D} 10 \mathrm{~d} \stackrel{\text { detachment space(reverse Higgs field) }}{\longrightarrow}$ massless particles

$\stackrel{\text { partial attachment space(Higgs field) }}{\longrightarrow}$ massive particles, massless particles, Higgs boson

4D10d particle was sliced into six different particles: 4D9d, 4D8d, 4D7d, 4D6d, $4 \mathrm{D} 5 \mathrm{~d}$, and $4 \mathrm{D} 4 \mathrm{~d}$ equally by mass. Baryonic matter is $4 \mathrm{D} 4 \mathrm{~d}$, while dark matter consisted of the other five types of particles $(4 \mathrm{D} 9 \mathrm{~d}, 4 \mathrm{D} 8 \mathrm{~d}, 4 \mathrm{D} 7 \mathrm{~d}, 4 \mathrm{D} 6 \mathrm{~d}$, and 4D5d) as follows.

$$
\begin{aligned}
& \text { 10D4d } \stackrel{\text { cosmic inflation }}{\longrightarrow} 4 \mathrm{D} 10 \mathrm{~d} \stackrel{\text { the slicing }}{\longrightarrow} \\
& \text { baryonic matter }(4 \mathrm{D} 4 \mathrm{~d})+\text { dark matter (4D5d, 4D6d, 4D7d, 4D8d, 4D9d) }
\end{aligned}
$$

As a result, the mass ratio of dark matter to baryonic matter is 5 to 1 . At observed $68.6 \%$ dark energy [15], the calculated values for baryonic matter and dark matter (with the $1: 5$ ratio) are $5.2 \%(=(100-68.6) / 6)$ and $26 \%(=5.2 \times 5)$, respectively, in good agreement with observed $4.9 \%$ and $26.5 \%$, respectively [15].

As described in Section 3.2.2., the space structure with both attachment space and detachment produces binary partition space $(1)_{n}(0)_{n}$ for wave-particle duality in quantum mechanics, miscible space $(1+0)_{n}$ for miscible mass-energy in relativity, and binary lattice space $\left(\begin{array}{ll}1 & 0\end{array}\right)_{n}$ for virtual particles in quantum field theory. Binary lattice space is derived from the slicing of mass dimensions by detachment space as described by Bounias and Krasnoholovets [29] who propose another explanation of the reduction of $>4 \mathrm{D}$ space-time into $4 \mathrm{D}$ space-time by slicing $>4 \mathrm{D}$ space-time into infinitely many $4 \mathrm{D}$ quantized units surrounding the $4 \mathrm{D}$ core particle. Such slicing of $>4 \mathrm{D}$ space-time is like slicing 3-space D object into 2-space D object in the way stated by Michel Bounias as follows: "You cannot put a pot into a sheet without changing the shape of the 2-D sheet into a 3-D dimensional packet. Only a 2-D slice of the pot could be a part of sheet". 4D10d particles emerge after the inflation. 10d mass dimension is sliced by detachment space into $9 \mathrm{~d}, 8 \mathrm{~d}, 7 \mathrm{~d}, 6 \mathrm{~d}, 5 \mathrm{~d}$, and $4 \mathrm{~d}$ plus the binary lattice space $\left(\begin{array}{ll}1 & 0\end{array}\right)_{n}$ for virtual particles in quantum field theory. For an example, the slicing of $10 \mathrm{~d}$ particle into $4 \mathrm{~d}$ particle is as follows. 


$$
1_{10} \stackrel{\text { slicing }}{\longrightarrow} 1_{4} \quad \sum_{\mathrm{d}=5}^{10}\left(\begin{array}{lll}
0_{4} & 1_{4}
\end{array}\right)_{n, \mathrm{~d}}
$$

10d particle $4 \mathrm{~d}$ core particle binary lattice space

where 1 is attachment space, 0 is detachment space, $1_{10}$ is $10 \mathrm{~d}$ particle, $1_{4}$ is $4 \mathrm{~d}$ particle, $\mathrm{d}$ is the mass dimension number of the dimension to be sliced, $n$ as the number of slices for each dimension, and $\left(0_{4} 1_{4}\right)_{n}$ is binary lattice space with repetitive units of alternative $4 \mathrm{~d}$ attachment space and $4 \mathrm{~d}$ detachment space. For $4 \mathrm{~d}$ particle starting from $10 \mathrm{~d}$ particle, the mass dimension number of the dimension to be sliced is from $d=5$ to $d=10$. Each mass dimension is sliced into infinite quantized units $(n=\infty)$ of binary lattice space, $\left(0_{4} 1_{4}\right)_{\infty}$. For $4 \mathrm{~d}$ particle, the $4 \mathrm{~d}$ core particle is surrounded by 6 types (from $\mathrm{d}=5$ to $\mathrm{d}=10$ ) of infinite quantized units of binary lattice space. Such infinite quantized units of binary lattice space represent the infinite units $(n=\infty)$ of separate virtual orbitals for virtual particles in a gauge force field, while the dimension to be sliced is "mass dimensional orbital" (DO), representing a type of gauge force field. In addition to the six DO's for gauge force fields from $d=5$ to $d=10$, the weak internal gravity appears as the seventh DO at $d=11$. As a result, there are seven mass dimensional orbitals as in Figure 3.

The seven mass dimensions are arranged as $\mathrm{F}_{5} \mathrm{~B}_{5} \mathrm{~F}_{6} \mathrm{~B}_{6} \mathrm{~F}_{7} \mathrm{~B}_{7} \mathrm{~F}_{8} \mathrm{~B}_{8} \mathrm{~F}_{9} \mathrm{~B}_{9} \mathrm{~F}_{10} \mathrm{~B}_{10}$ $F_{11} B_{11}$, where $F_{d}$ and $B_{d}$ are mass dimensional fermion and mass dimensional boson, respectively. Under the varying supersymmetry dimensional (VSD) transformation, the mass of mass dimensional fermion and the mass of mass dimensional boson are related to each other with three simple formulas as the follows.

$$
\begin{gathered}
M_{\mathrm{d}, \mathrm{B}}=M_{\mathrm{d}, \mathrm{F}} / \alpha_{\mathrm{d}} \\
M_{\mathrm{d}+1, \mathrm{~F}}=M_{\mathrm{d}, \mathrm{B}} / \alpha_{\mathrm{d}+1} \\
M_{\mathrm{d}+1, \mathrm{~B}}=M_{\mathrm{d}, \mathrm{B}} / \alpha_{\mathrm{d}+1}^{2},
\end{gathered}
$$

where $\mathrm{d}$ is the mass dimension number, $\mathrm{F}$ is fermion, and $\mathrm{B}$ is boson. Each dimension has its own $\alpha_{\mathrm{d}}$, and all $\alpha_{\mathrm{d}}$ 's except $\alpha_{7}\left(\alpha_{w}\right)$ of the seventh dimension (weak interaction) are equal to $\alpha$, the fine structure constant of electromagnetism.

As shown in the previous paper "Split Membrane 11D Spacetime $=1 \mathrm{D}$ Eleventh Dimension Interval Space $+6 \mathrm{D}$ Rishon Space $+3 \mathrm{D}$ Higgs Space $+1 \mathrm{D}$ Einstein Time: Cosmology" [10], quarks are derived from the split $11 \mathrm{D}$ membrane. To represent quarks, the additional seven mass dimensions as the "auxiliary mass dimensions" (a's) to represent quarks and unstable leptons in addition to the seven "principal mass dimensions" (d's) to represent neutrino-electron and gauge bosons as in Figure 4.

\section{The Periodic Table of Elementary Particles}

The periodic table of elementary particles [25] [30] is based on the seven principal mass dimensions (d's) for stable baryonic matter leptons (electron and neutrinos), gauge bosons (all forces), gravity, and dark matter (five sterile dark 




Figure 3. The seven mass dimensions as mass dimensional orbitals.

Stable Baryonic Matter Leptons

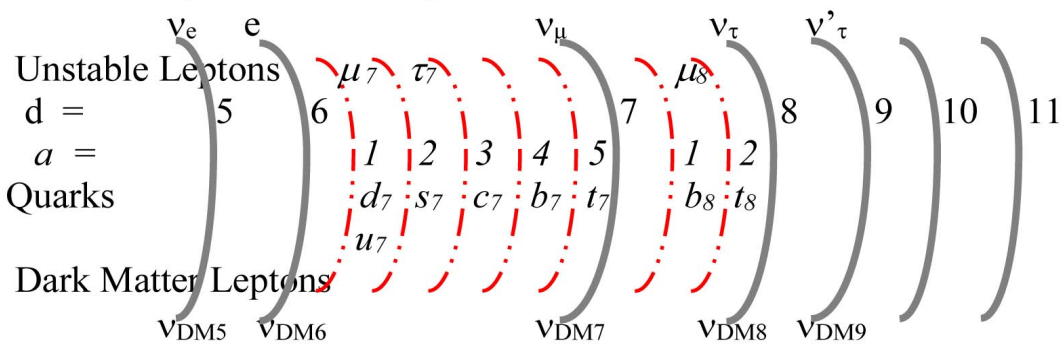

Figure 4. The seven principal mass dimensions (solid lines) denoted by the principal mass dimension number $d$ and the seven auxiliary mass dimensions (dash-dotted lines) denoted by the auxiliary mass dimension number $a$.

matter neutrinos) and the seven auxiliary mass dimensions (a's) for unstable leptons (muon and tau) and quarks (d, u, s, c, b, and t) as in Figure 4 and Table 1 .

In the periodic table of elementary particles, the five dark matter particles are derived from Equation (33). Without electromagnetism at $d=5$, dark matter does not have charge particle, and has to be neutrinos. Initially derived from Equation (33) and the symmetry between dark matter and baryonic matter, there were five dark matter massive right-handed neutrinos and one baryonic matter massive left-handed neutrino. Through the reverse Higgs mechanism as Equation (27), the left-handed neutrino becomes massless, while the right-handed neutrinos as sterile dark matter neutrinos remain massive. The reverse Higgs boson was observed [24].

As sterile neutrinos, dark matter does not react with baryonic matter possibly except baryonic neutrinos. A new observation of Excess Electronic Recoil Events in XENON1T indicates an excess over known backgrounds is observed below 7 $\mathrm{keV}$, rising towards lower energies and prominent between $2-3 \mathrm{keV}$ which enables the most sensitive searches for solar axions, an enhanced neutrino magnetic moment using solar neutrinos, and bosonic dark matter [31]. This event can be the rare reaction between massive $v_{\mathrm{DM}}$ and massless $v_{\mathrm{BM}}$ to produce boson $\mathrm{B}_{5}$ at $3.7 \mathrm{keV}$ (Table 2).

$$
\text { massive } v_{\mathrm{DM}}+\text { massless } \bar{v}_{\mathrm{BM}} \rightarrow \text { boson } \mathrm{B}_{5} \text { at } 3.7 \mathrm{keV}
$$

All neutrinos and electron as well as gauge bosons are in the principal mass dimensions. All quarks and unstable leptons are in the auxiliary mass dimensions. The three generations of baryonic matter lepton-quark is the maximum generations allowed for the seven principal dimensions and the seven auxiliary dimensions. 
Table 1. The periodic table of elementary particles for baryonic matter and dark matter. $\mathrm{d}$ $=$ principal mass dimension number, $\mathrm{a}=$ auxiliary mass dimension number, $\mathrm{DM}=$ dark matter, $\mathrm{BM}=$ baryonic matter.

\begin{tabular}{|c|c|c|c|c|c|c|c|c|}
\hline d & $a=0$ & $a=0$ & 1 & 2 & 1 & 23 & $\begin{array}{lll}3 & 4 & 5\end{array}$ & $a=0$ \\
\hline & $\frac{\text { Stable Baryonic }}{\text { Matter Leptons }}$ & $\frac{\text { Dark Matter }}{\underline{\text { Leptons }}}$ & $\begin{array}{l}\text { Unstabl } \\
\text { Leptons }\end{array}$ & & & Quarks & & $\underline{\text { Bosons }}$ \\
\hline 5 & $v_{\mathrm{e}}$ & $v_{\mathrm{DM} 5}$ & & & & & & $\mathrm{~B}_{5}=\mathrm{A}$ electromagnetism \\
\hline 6 & e & $v_{\mathrm{DM} 6}$ & & & & & & $\begin{array}{c}\mathrm{B}_{6}=\mathrm{g}^{*} \text { strong (basic } \\
\text { gluon for quarks) }\end{array}$ \\
\hline 7 & $v_{\mu}$ & $v_{\mathrm{DM} 7}$ & $\mu_{7}$ & $\tau_{7}$ & $\mathrm{~d}_{7} / u_{7}$ & $\mathrm{~s}_{7} \quad \mathrm{c}$ & $c_{7} \quad b_{7} t_{7}$ & $\begin{array}{c}\mathrm{B}_{7}=\mathrm{Z}_{\mathrm{L}}^{0} \text { left-handed BM } \\
\text { weak }\end{array}$ \\
\hline 8 & $v_{\tau}$ & $v_{\mathrm{DM} 8}$ & $\begin{array}{c}\mu_{8} \\
\text { (absent) }\end{array}$ & & $\begin{array}{c}\mathrm{b}_{8} \\
\text { (absent) }\end{array}$ & $\mathrm{t}_{8}$ & & $\begin{array}{c}\mathrm{B}_{8}=\mathrm{Z}_{\mathrm{R}}^{0} \text { right-handed DM } \\
\text { weak }\end{array}$ \\
\hline 9 & $v_{\tau}^{\prime}\left(\right.$ high-mass $\left.v_{\tau}\right)$ & $v_{\mathrm{DM} 9}$ & & & & & & $\begin{array}{c}\mathrm{B}_{9}=\text { dark matter repulsive } \\
\text { force }\end{array}$ \\
\hline 10 & & & & & & & & $\begin{array}{c}\mathrm{B}_{10}=\text { particle-antiparticle } \\
\text { asymmetry }\end{array}$ \\
\hline 11 & gravitino & & & & & & & $\mathrm{B}_{11}=$ gravity \\
\hline
\end{tabular}

Table 2. The masses of the principal mass dimensions (gauge bosons).

\begin{tabular}{ccccc}
\hline $\mathrm{B}_{\mathrm{d}}$ & $M_{\mathrm{d}}$ & $\mathrm{GeV}$ (calculated) & Gauge boson & Interaction \\
\hline $\mathrm{B}_{5}$ & $M_{\mathrm{e}} \alpha$ & $3.7 \times 10^{-6}$ & $\mathrm{~A}=$ photon & Electromagnetic \\
$\mathrm{B}_{6}$ & $M_{\mathrm{e}} / \alpha$ & $7 \times 10^{-2}(70.02 \mathrm{MeV})$ & $\mathrm{g}^{*}=$ basic gluon & Strong \\
$\mathrm{B}_{7}$ & $M_{\mathrm{Z}}=M_{\mathrm{B} 6} / \alpha_{w}^{2}$ & 91.1876 (given) & $\mathrm{Z}_{\mathrm{L}}$ & weak (left) for baryonic matter \\
$\mathrm{B}_{8}$ & $M_{7} / \alpha^{2}=M_{\mathrm{Z}} / \alpha^{2}$ & $1.71 \times 10^{6}$ & $\mathrm{Z}_{\mathrm{R}}$ & weak (right) for dark matter \\
$\mathrm{B}_{9}$ & $M_{8} / \alpha^{2}=M_{\mathrm{Z}} / \alpha^{4}$ & $3.22 \times 10^{10}$ & & dark matter repulsive force \\
$\mathrm{B}_{10}$ & $M_{9} / \alpha^{2}=M_{\mathrm{Z}} / \alpha^{6}$ & $6.04 \times 10^{14}$ & & particle-antiparticle asymmetry \\
$\mathrm{B}_{11}$ & $M_{10} / \alpha^{2}=M_{\mathrm{Z}} / \alpha^{8}$ & $1.13 \times 10^{19}$ & $\mathrm{G}$ & gravity \\
\hline
\end{tabular}

\section{Gauge Bosons}

In the periodic table of elementary particles, the given observed masses are the mass of electron for $\mathrm{F}_{6}$ and the mass of $\mathrm{Z}$ boson for $\mathrm{B}_{7}$. From Equations (35), (36) and (37), $\alpha_{w}=\alpha_{7}=\alpha$ of week interaction $=\left(M_{\mathrm{B} 6} / M_{\mathrm{B} 7}\right)^{1 / 2}=\left(M_{\mathrm{F} 6} / \alpha / M_{\mathrm{B} 7}\right)^{1 / 2}=$ $\left(M_{\mathrm{e}} / \alpha / M_{\mathrm{Z}}\right)^{1 / 2}=0.02771$. Therefore, the masses of gauge bosons are as in Table 2.

The lowest energy gauge boson $\left(B_{5}\right)$ at $d=5$ is the Coulomb field for electromagnetism. The second gauge lowest boson $\left(B_{6}\right)$ at $d=6$ is basic gluon $\left(g^{\star}=70\right.$ $\mathrm{MeV} \approx$ one half of pion) is the strong force as the nuclear force in the pion theory [32] where pions mediate the strong interaction at long enough distances (longer than the nucleon radius) or low enough energies. $\mathrm{B}_{6}$ is denoted as basic gluon, $g^{\star}$. At short enough distances (shorter than the nucleon radius) or high enough energies, gluons emerge to confine fractional charge quarks. Fractional charge quarks are confined by gluons in QCD (quantum chromodynamics). No 
isolated fractional charge quark is allowed, and only collective integer charge quark composites are allowed. In general, collective fractional charges are confined by the short-distance confinement force field where the sum of the collective fractional charges is integer [33]. As a result, fractional charges are confined and collective. The confinement force field includes gluons for collective fractional charge quarks in hadrons and the magnetic flux quanta for collective fractional charge quasiparticles in the fractional quantum Hall effect (FQHE) [34] [35] [36].

The third lowest boson $\left(B_{7}\right)$ at $d=7$ is $Z_{L}$ for the left-handed weak interaction among leptons and quarks. Spontaneous symmetry breaking produces massive weak bosons, massless photon and the Higgs boson as Equation (27). Massive weak bosons produce short-distance interaction. $B_{8}$ at $d=8$ is $Z_{R}$ for the right-handed weak interaction among dark matter neutrinos as dark matter neutrino oscillation. The symmetry between $Z_{R}$ and $Z_{L}$ provides the neutrino oscillation for both baryonic matter neutrinos [37] and dark matter neutrinos.

$\mathrm{B}_{9}$ as the gauge boson represents dark matter repulsive force. The condensed baryonic gas at the critical surface density (derived from the acceleration constant $\mathrm{a}_{0}$ in MOND [13] [14]) induces the creation tensor for dark matter repulsive force to transform dark matter in the region into repulsive dark matter repulsing one another, corresponding to the Farnes' repulsive dark matter. Before the emergence of dark matter repulsive force, dark matter in the CMB was not repulsive.

$B_{10}$ at $d=10$ is for the gauge boson for particle-antiparticle asymmetry to provide the slight excess of particle in particle-antiparticle at the Big Bang, while $\mathrm{B}_{8}$ has particle-antiparticle symmetry. ( $\mathrm{B}_{9}$ emerged long after the Big Bang.) As a result, the excess of particle is $\alpha^{4}\left(2.8 \times 10^{-9}\right)$ per particle-antiparticle (photon) for the ratio between $B_{8}$ and $B_{10}$. Since baryonic matter is $1 / 6$ of dark matter and baryonic matter from Equation (33), the baryonic matter excess is $4.7 \times 10^{-10}$ which is in a good agreement with $6 \times 10^{-10}$ for the ratio of the numbers between baryonic matter and photons in the Big Bang nucleosynthesis [38].

$B_{11}$ is for gravity. $F_{11}\left(8.275 \times 10^{16} \mathrm{GeV}\right)$ relates to spin $3 / 2$ gravitino, while $B_{11}$ $\left(1.134 \times 10^{19} \mathrm{GeV}\right)$ relates to spin 2 graviton. In supersymmetry, gravitino and graviton mediate the supersymmetry between fermion and boson in space dimension and gravitation. There are 11 space dimensions in the 11 spacetime dimensional membrane. As a result, the supersymmetry involves $11 \mathrm{~F}_{11}+\mathrm{B}_{11}$, which is equal to $1.225 \times 10^{19} \mathrm{GeV}$ in excellent agreement with the Planck mass $\left(1.221 \times 10^{19} \mathrm{GeV}\right)$ derived from observed gravity as $(\hbar c / G)^{1 / 2}$ where $c$ is the speed of light, $G$ is the gravitational constant, and $\hbar$ is the reduced Planck constant.

\section{Leptons and Quarks}

The seven dimensional orbitals are the base for the periodic table of elementary particles [39] [40] to produce the standard model particles and the internal gravity. The periodic table of elementary particles calculates accurately the par- 
ticle masses of all leptons, quarks, gauge bosons, the Higgs boson, and the cosmic rays by using only five known constants: the number (seven) of the extra spatial dimensions in the eleven-dimensional membrane, the mass of electron, the masses of $\mathrm{Z}$ and $\mathrm{W}$ bosons, and the fine structure constant [39]. The calculated masses are in excellent agreements with the observed masses. For examples, the calculated masses of muon, top quark, pion, neutron, and the Higgs boson are $105.55 \mathrm{MeV}, 175.4 \mathrm{GeV}, 139.54 \mathrm{MeV}, 939.43 \mathrm{MeV}$, and $126 \mathrm{GeV}$, respectively, in excellent agreements with the observed $105.65 \mathrm{MeV}, 172.4 \mathrm{GeV}, 139.57$ $\mathrm{MeV}, 939.27 \mathrm{MeV}$, and $126 \mathrm{GeV}$, respectively.

4b) the formation of the hidden oscillating dimension number universes from $10 \mathrm{D}$ to $5 \mathrm{D}$

The negative-mass 10D particle-antiparticle, the positive-mass external gravity, and the negative-mass external gravity are transformed into the hidden oscillating dimension number universes from 10D to 5D.

$$
\begin{aligned}
& 10 \mathrm{D} 4 \mathrm{~d} \rightarrow \text { 9D5d } \rightarrow \text { 9D4d } \rightarrow \text { 8D } 5 \mathrm{~d} \rightarrow \text { 8D } 4 \mathrm{~d} \rightarrow 7 \mathrm{D} 5 \mathrm{~d} \\
& \rightarrow 7 \mathrm{D} 4 \mathrm{~d} \rightarrow 6 \mathrm{D} 5 \mathrm{~d} \rightarrow 6 \mathrm{D} 4 \mathrm{~d} \rightarrow 5 \mathrm{D} 5 \mathrm{~d} \rightarrow 5 \mathrm{D} 4 \mathrm{~d}
\end{aligned}
$$

From Equation (19), under the VSLD transformation and the VSD transformation, the three universes expand through the increasing rest mass and the translation-fractionalization from $10 \mathrm{D} 4 \mathrm{~d}$ to $5 \mathrm{D} 4 \mathrm{~d}$. To the positive-mass $4 \mathrm{D}$ universe, the three universes from $10 \mathrm{D}$ to $5 \mathrm{D}$ are hidden, because as mentioned in Section 3.1.2., particles with different space-time dimensions and different speeds of light are transparent and oblivious to one another to avoid the violation of causality due to differences in the speed of light. During this time, the positive-mass $4 \mathrm{D}$ universe expands normally.

5) the transformation of all four universes into the 4D universes

When all four universes become $4 \mathrm{D}$, the three other universes become dark energy as a part of the positive-mass $4 \mathrm{D}$ universe.

$$
5 \mathrm{D} 4 \mathrm{~d} \rightarrow 4 \mathrm{D} 5 \mathrm{~d} \rightarrow \text { 4D4d }
$$

The result is the accelerating expansion. Since the other three universes have no detachment space to produce kinetic energy, dark energy is inert as the inert cosmological constant. As these three universes can take the form of a cosmological constant, the field equations are modified to

$$
R_{\mu v}-\frac{1}{2} \operatorname{Rg}_{\mu v}+\Lambda g_{\mu v}=\frac{8 \pi G}{c^{4}}\left(T_{\mu \nu}^{+}+T_{\mu \nu}^{-}+C_{\mu \nu}\right)
$$

where $R_{\mu \nu}$ is the Ricci curvature tensor, $R$ is the scalar curvature, $g_{\mu \nu}$ is the metric tensor, $\Lambda$ is the cosmological constant, $G$ is Newton's gravitational constant, $c$ is the speed of light in vacuum, $T_{\mu \nu}^{+}$is the positive stress-energy tensor for the positive-mass universe, $T_{\mu \nu}^{-}$is the positive-negative stress-energy tensor for negative-mass universe, negative-mass external gravity, and positive-mass external gravity, and $C_{\mu \nu}$ is the creation tensor for negative-mass universe, negative-mass external gravity, and positive-mass external gravity.

The ratio of the time periods for the transformations from $\mathrm{D} \rightarrow \mathrm{D}-1$ is pro- 
portional to ln of the total number of particles (Equation (22)) to be transformed from $\mathrm{D} \rightarrow \mathrm{D}-1$ for the exponential growth with time as in Table 3.

The maximum dark energy is $75 \%$ for the three out of the four universes, when the spacetime numbers of all particles are 4 . The observed $\%$ of dark energy is 68.6 , and our universe is 13.8 billion-year old [15]. The period of the $5 \mathrm{D} \rightarrow$ $4 \mathrm{D}$ is $(0.333)(13.8) /((0.333)(68.6 / 75)+0.667)=4.73$ billion years, and dark energy as the $5 \mathrm{D} \rightarrow 4 \mathrm{D}$ started in $(4.73)(68.6 / 75)=4.33$ billon years ago that is in agreement with the observed value of $4.71 \pm 0.98$ billion years ago when dark energy started to accelerate the cosmic expansion [16].

6) the positive-mass $4 \mathrm{D}$ universe and the three hidden oscillating dimension number universes from $5 \mathrm{D}$ to $9 \mathrm{D}$

The three oscillating universes from $5 \mathrm{D}$ to $10 \mathrm{D}$ again become the hidden universes.

$$
\begin{aligned}
& \text { 4D4d } \rightarrow \text { 5D4d } \rightarrow \text { 5D5d } \rightarrow \text { 6D4d } \rightarrow \text { 6D5d } \rightarrow \text { 7D4d } \rightarrow \text { 7D5d } \\
& \rightarrow \text { 8D4d } \rightarrow \text { 8D5d } \rightarrow \text { 9D4d } \rightarrow \text { 9D5d }
\end{aligned}
$$

They contract by the decreasing rest mass and the translation-condensation. The positive-mass $4 \mathrm{D}$ universe contracts through gravity. Through symmetry, all four universes contract synchronically and equally.

7) the return to the 10D4d particle-antiparticle universes (step 3)

Eventually, the oscillating universes return to the original 10D. The positive-mass $4 \mathrm{D}$ universe reaches the Big Crush to lose all detachment space to become 4D10d, and followed by the deflation to transform into 10D4d. The four universes return to the step 3 .

In the positive-energy $4 \mathrm{D}$ universe

$4 \mathrm{D}+$ various d's particles $\stackrel{\text { the Big Crush }}{\longrightarrow} 4 \mathrm{D} 10 \mathrm{~d}$ particles $\stackrel{\text { the deflation }}{\longrightarrow} 10 \mathrm{D} 4 \mathrm{~d}$ particles In the other three universes

9D5d particles $\stackrel{\text { VSLD }}{\longrightarrow} 10 \mathrm{D} 4 \mathrm{~d}$ particles

From the step 3, the universes can undergo another cycle of the particle-antiparticle universes, or can reverse to the step 2 for the string-antistring dual universes, to the step 1 for the membrane-antimembrane dual universes, and ultimately, to the zero-mass inter-universal void.

\section{Protogalaxy and Galaxy Evolutions}

As mentioned in the previous section, with positive mass dark matter, the Navarro-

Table 3. The Percentages of the periods in the oscillating dimension number universes. $\alpha$ is the fine structure constant for electromagnetism.

\begin{tabular}{ccccccc}
\hline & $10 \mathrm{D} \rightarrow 9 \mathrm{D}$ & $9 \mathrm{D} \rightarrow 8 \mathrm{D}$ & $8 \mathrm{D} \rightarrow 7 \mathrm{D}$ & $7 \mathrm{D} \rightarrow 6 \mathrm{D}$ & $6 \mathrm{D} \rightarrow 5 \mathrm{D}$ & $5 \mathrm{D} \rightarrow 4 \mathrm{D}$ \\
\hline ratio of total numbers of particles & 1 & $\alpha^{-2}$ & $\alpha^{-4}$ & $\alpha^{-6}$ & $\alpha^{-8}$ & $\alpha^{-10}$ \\
ratio of $\ln$ (total number of particles) & 0 & $-2 \ln \alpha$ & $-4 \ln \alpha$ & $-6 \ln \alpha$ & $-8 \ln \alpha$ & $-10 \ln \alpha$ \\
ratio of periods in time & $\sim 0$ & 1 & 2 & 3 & 4 & 5 \\
percentages of periods in time & $\sim 0$ & 6.7 & 13.3 & 20 & 26.7 & 33.3 \\
\hline
\end{tabular}


Frenk-White (NFW) profile [2] derived from standard N-body simulations has the high density dark matter at the core of galaxy, but the high density dark matter at the core of galaxy is not observed [3]. This is known as the core-cusp problem or the cuspy halo problem and is currently unsolved with positive mass dark matter [17].

Another unsolved problem is central supermassive black hole ( $\mathrm{SMBH})$ that is located in most massive galaxies. Its origin remains unclear because the greatest difficulty to any theory of SMBH formation has been the observation of very massive $\left(M \approx 10^{9} \mathrm{M}_{\odot}\right)$ quasars with $\mathrm{SMBH}$ already in place by $\mathrm{z} \approx 7$, when the universe is just $\approx 800 \mathrm{Myr}$ old long before any stars could grow big or old enough to collapse under their own mass, explode in a supernova and form a black hole [41]. Considerable observational and theoretical evidence shows the viability of massive black hole seeds, formed by the collapse of supermassive stars (SMSs) as a progenitor model for such early, massive accreting black holes [42]. However, it is unclear how such a large SMS remained stable enough without first forming many large stars.

A new unsolved problem is the recent discovery of a cold, massive, rotating disk galaxy (the Wolfe Disk) 1.5 billion years after the Big Bang [43]. Massive disk galaxies like the Milky Way are expected to form at late times in traditional models of galaxy formation [44]. Recent numerical simulations suggest that such galaxies with billion solar masses could form as early as a billion years after the Big Bang through the accretion and merger of cold material [45], but it is not clear how to assemble such a large gas mass while maintaining a relatively stable, rotating disk without breaking into many small galaxies. The Wolfe Disk's large rotational velocity and large content of cold gas remain challenging to reproduce with most numerical simulations.

In this paper, dark matter with and without repulsive force provides an answer to all three unsolved problems, and explains protogalaxy evolution and galaxy evolution. This paper proposes that the condensed baryonic gas at the critical surface density induces the creation tensor for dark matter repulsive force to transform dark matter in the region into repulsive dark matter repulsing one another. Repulsive dark matter removes the DM (dark matter) core-cusp, and provides stabilities for SMS and large protogalaxy before the formation of a large galaxy. Without repulsive dark matter, the universe would have been filled with "train wrecks" without large galaxy structures.

\subsection{Protogalaxy Evolution}

Protogalaxy evolution consists of six steps: 1) Small Core-Cusp Protogalaxy (BM-DM core cusp-halo), 2) Large Core-Cusp Protogalaxy (BM-DM core cusp-halo), 3) Streaming Protogalaxy (streaming BM core-static BM shell-DM halo), 4) SMBH Protogalaxy (central SMBH-streaming BM core-static BM shell-DM halo). 5) Bulge Protogalaxy (central SMBH-bulge-streaming BM core-static BM shell-DM halo), and 6. Protogalaxy Termination (central SMBH-bulge-combined BM shell-DM halo) as Figure 5. 


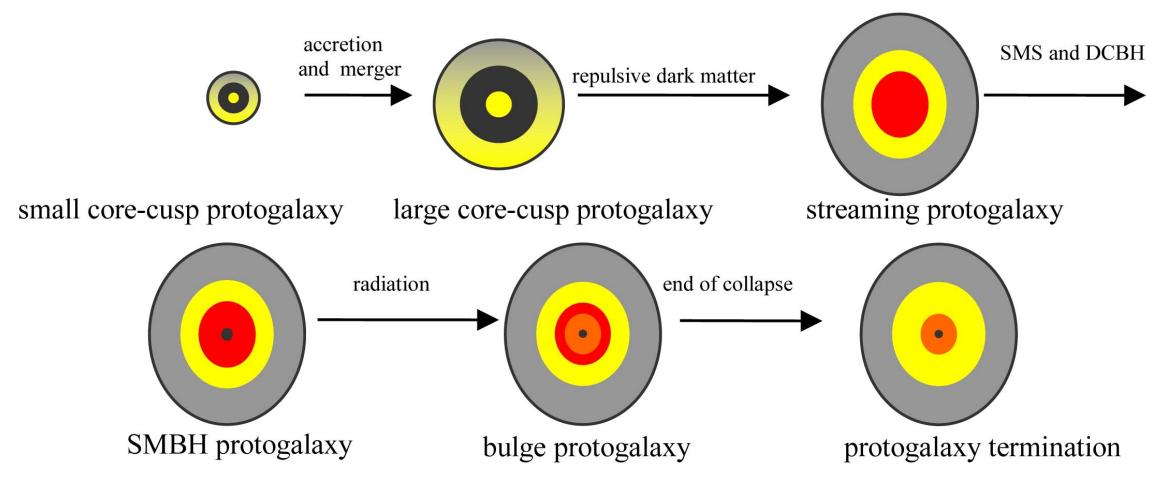

Figure 5. Protogalaxy Evolution: 1) small core-cusp protogalaxy (BM-DM core cusp-halo), 2) large core-cusp protogalaxy (BM-DM core cusp-halo), 3) streaming protogalaxy (streaming BM core-static BM shell-DM halo), 4) SMBH protogalaxy (central SMBH-streaming BM core-static BM shell-DM halo). 5) bulge protogalaxy (central SMBH-bulge-streaming BM core-static BM shell-DM halo), and 6) protogalaxy termination (central SMBH-bulgecombined BM shell-DM halo).

\section{1) The Small Core-Cusp Protogalaxy Step}

Quantum fluctuations in the matter distribution were created in the first fraction of a second during an inflationary period. Gravitational instability grew these fluctuations over time. Baryonic matter and dark matter without dark matter repulsive force were initially well mixed. Dark halos emerged as gravitationally bound regions of matter that have decoupled from the cosmic expansion and collapsed. Dark matter haloes were formed as in N-body collisionless dark matter simulations. They followed the NFW profile where the highest density is at the center as the core cusp [2]. Baryonic matter was dragged along by the gravitationally dominant dark matter with the highest density at the center, resulting in small BM-DM core-cusp-halo.

\section{2) The Large Core-Cusp Protogalaxy Step}

Dark matter halo structure formation is bottom-up merge with small dark matter haloes forming first. The baryonic gas content can contract together with the dark matter only in dark halos above the cosmological Jeans mass, $\mathrm{MJ} \approx 10^{4}$ $\mathrm{M}_{\odot}[(1+\mathrm{z}) / 11]^{3 / 2}$, in which the gravity of dark matter can overwhelm thermal gas pressure [46]. At $\mathrm{z} \sim 1100$ (400 thousand years old), the temperature drops below $\sim 3000 \mathrm{~K}$ and protons and electrons recombine to form neutral hydrogen. The photons then decouple and travel freely until the present, when they are observed as the CMB (cosmic microwave background) [47] [48]. Following recombination and cooling through the emission of radiation, the neutral gas was freed to fall into the gravitational potential wells of the dark matter, and to condense into the BM core. Unable to dissipate radiation and condense, dark matter stayed outside the BM core to form the DM core cusp-halo, resulting in the large core-cusp protogalaxy consisting of large BM-DM core cusp-halo [49].

\section{3) The Streaming Protogalaxy Step}

The condensed baryonic gas at the critical surface density (derived from the acceleration constant $a_{0}$ in MOND [13] [14]) induces the creation tensor for 
dark matter repulsive force to transform dark matter in the region into repulsive dark matter repulsing one another, corresponding to the Farnes' repulsive dark matter. Dark matter repulsive force in the periodic table of elementary particles for baryonic matter and dark matter (Table 1) is a force like the other forces, such as the strong, the weak, electromagnetic, and gravitational forces. The repulsive dark matter particles with dark matter repulsive force in the DM core cusp stream outward to leave a low density dark matter core and to form a halo that surrounds the positive mass galaxy and which extends out to several galactic radii as in the Farnes' unifying theory for repulsive dark matter. The result is the protogalaxy collapse which is like the collapse of a balloon as the air (as dark matter) moving out the balloon. The monolithic protogalaxy collapse forced baryons in the halo region to stream inward to fill the low density core. The result was the streaming protogalaxy with the streaming BM core-static BM shell-DM halo. In some cases, the ejected streaming dark matter jet inevitably carried some baryonic matter out of the protogalaxy. The baryonic matter in the ejected dark matter jet resulted eventually in the dwarf galaxies which are observed as satellite galaxies arranged on a plane as observed by Ibata et al. [50].

4) The SMBH Protogalaxy Step

Primordial halos expose to highly supersonic baryon streaming motions [51] [52] which can prevent protogalaxies from forming stars, so the streaming protogalaxies remained stable until they reach masses of $10^{7}-10^{8} \mathrm{M}_{\odot}$ that trigger rapid atomic cooling and catastrophic baryon collapse to start forming SMSs at central infall rates of up to $\approx 1 \mathrm{M}_{\odot} \mathrm{yr}^{-1}$ in the atomically cooled protogalaxies [53] [54]. The collapse of the SMS at a few $10^{5} \mathrm{M}_{\odot}$ undergoes direct collapse black hole (DCBH) without supernova to produce the central $\mathrm{SMBH}$ [55] [56] [57]. The DCBH era lasts from $\mathrm{z} \approx 20$ to $\mathrm{z} \approx 13$, or a time period $\mathrm{T} \approx 150$ Myr. The whole DCBH process stops, when the gas in the atomically cooled halos is photoevaporated by the ambient radiation field [58]. According to the mathematical model of the growth of DCBH in the early universe by Basu and Das [59], the mass growth function of DCBH gains an exponentially large amount of mass in a relatively short amount of time. The rapid growth is capped by the Eddington limit, and for a brief period of time exceeds the Eddington limit before the termination of the DCBH growth. The protogalaxy collapse prevented the fragmentation of baryons during the formation of the SMS as all fragments merged in the core. The result was the SMBH protogalaxy with the SMBH-streaming BM core-static BM shell-DM halo.

\section{5) The Bulge Protogalaxy Step}

The incoming streaming baryons during the protogalaxy collapse accelerated the growth of the SMBH. Eventually, the radiation from the SMBH reduced greatly the streaming motion of the incoming streaming baryons, and the SMBH was decoupled from the streaming BM core as described by Joseph Silk, Martin Rees, and Andrew King [60] [61]. Without strong streaming, baryons were fragmented to form normal stars, resulting in the bulge in the streaming BM 
core. The result was the bulge protogalaxy with the SMBH-bulge-streaming BM core-static BM shell-DM halo. Since the SMBH and the bulge were formed in the streaming $\mathrm{BH}$ core, the mass of the $\mathrm{SMBH}$ and the mass of bulge are correlated as found in the studies [62] [63].

6) The Protogalaxy Termination Step

Eventually, without streaming, the streaming BM core and the static BM shell were combined into the combined region, resulting in the SMBH-bulge-combined BM shell (streaming BM core + the static BM shell)-DM halo. The protogalaxy termination means the end of the protogalaxy collapse. The protogalaxy termination step started galaxy evolution when the star formation in the combined region in a large galaxy became active.

\subsection{Galaxy Evolution}

Elliptical galaxies are mildly flattened, and are mainly supported by the random motions of their stars. Spiral galaxies, on the other hand, have highly flattened disks that are mainly supported by rotation of their stars. Most elliptical and spiral galaxies have both with the ellipsoidal component called the bulge. Irregular galaxies do not have definite shape and the bulge. In this paper, the morphology of galaxies is derived from the monolithic heterogeneous protogalaxy collapse which produced both SMBH and galaxies. Essentially as described in this section, the protogalaxies with the small DM core cusp produced elliptical galaxies, the protogalaxies with the large DM core cusp produced spiral galaxies, and the protogalaxies with the very large DM core cusp produced irregular galaxies.

1) The Formation of Elliptical Galaxy

The progenitor of elliptical galaxy is the protogalaxy with small DM core cusp and large BM shell. The ejection of dark matter from a small DM core cusp resulted in a small empty core which was filled by the streaming baryons to form a small streaming BM core surrounded by a large static BM shell. In terms of star formation, the normal star formation took place only in the large BM shell, while the SMS and DCBH formation took place only in the small streaming BM core. The large amount of baryon gas was depleted by the star formation in the large static BM shell. The stars are in random orbits around the center. The protogalaxy collapse caused the slight flattering of the protogalaxy into elliptical shape whose lengths of major axes are proportional to the relative sizes of the DM core cusp. The early large consumption of gas by the star formation in the large static BM shell depleted large amount of baryon gas, so elliptical galaxies now have very few young stars.

During the early universe, large protogalaxies accreted the surrounding small and medium protogalaxies, and turned them into metal-poor globular clusters without external dark matter haloes [64] in galactic halos. (Some of the accreted protogalaxies had their own accreted protogalaxies.) As a result, the stars in globular clusters in general are older than the stars in the host large galaxies. 


\section{2) The Formation of Spiral Galaxy and Barred Spiral Galaxy}

The progenitor of spiral galaxy is the protogalaxy with large DM core cusp and small BM shell. The ejection of dark matter from a large DM core cusp resulted in a large empty core which was filled by the streaming baryons to form a large streaming BM core surrounded by a small static BM shell. During the protogalaxy collapse, the streaming baryons initially formed a dense cloud at the center of the streaming BM core. The infalling streaming baryons toward the dense cloud at the center produced the rotational baryon cloud due to the conservation of angular momentum, similar to the rotational cloud during the collapse of the cloud. Eventually, the whole streaming BM core and then the whole protogalaxy became rotational. (In the progenitor of elliptical galaxy, the streaming BM core was too small to rotate the whole protogalaxy.) The stars orbit around the center.

After the termination of protogalaxy collapse, the collapsed protogalaxy consisted of central SMBH-bulge-combined BM shell-DM halo. After the end of the protogalaxy, the protogalaxy underwent differential rotation with the increasing angular speeds toward the center. After few rotations, the protogalaxy turned into the spiral structure consisting of the center disk with the pre-existed bulge-SMBH at the center and the attached spiral arms. The arms consisted of the low density gas regions and the high density gas regions. The high density gas regions hindered the rotational movement of the low baryon gas, so the low baryon gas formed the large high density regions behind the high density gas regions. After few rotations, all large high density regions coalescent into few major arms at the minimum rotational velocities as the high density waves described in the density wave theory by C. C. Lin and Frank Shu [65] [66]. The arms in spiral galaxy are the high density waves. Near the center of a spiral galaxy, the high density wave forms the bar for barred spiral galaxy [67]. The evolution of spiral and barred spiral galaxies is shown in Figure 6.

Without the early large depletion of baryon gas in the small BM shell and with the radiation from $\mathrm{SMBH}$ and stars to retard the star formation rate, spiral galaxies



Figure 6. The formations of spiral galaxy and barred spiral galaxy. 
and barred spiral galaxies now have many young stars and large amounts of interstellar gas. The stars form in the spiral arms much later than in the bulge and the bar, so they are many young stars in the spiral arms. The lengths of the spiral arms are proportional to the relative sizes of the DM core cusp as the lengths of major axes proportional to the relative sizes of the DM core cusp in elliptical galaxies.

3) The Formation of Irregular Galaxy

If the size of the DM core cusp was very large, the streaming dark matter in the DM core cusp streamed outward, and not enough baryonic matter particles from the BM-DM halo streamed inward to form SMS. Eventually, the protogalaxy became fragmented, resulting in irregular galaxy.

\section{Summary and Conclusion}

In summary, this paper modifies the Farnes' unifying theory of dark energy and dark matter which are negative-mass to be created continuously from the negative-mass universe in the positive-negative mass universe pair. The first modification explains that observed dark energy is $68.6 \%$ greater than $50 \%$ for the symmetrical positive-negative mass universe pair. This paper starts with the proposed positive-negative-mass 11D universe pair (without kinetic energy) which is transformed into the positive-negative mass 10D universe pair and the external dual gravities as in the Randall-Sundrum model, resulting in the four equal and separate universes consisting of the positive-mass 10D universe, the positive-mass massive external gravity, the negative-mass 10D universe, and the negative-mass massive external gravity. The positive-mass 10D universe is transformed into $4 \mathrm{D}$ universe (home universe) with kinetic energy through the inflation and the Big Bang to create positive-mass dark matter which is five times of positive-mass baryonic matter. The other three universes without kinetic energy oscillate between 10D and 10D through $4 \mathrm{D}$, resulting in the hidden universes when $\mathrm{D}>4$ and dark energy when $\mathrm{D}=4$, which is created continuously to our $4 \mathrm{D}$ home universe with the maximum dark energy $=3 / 4=$ $75 \%$. In the second modification to explain dark matter in the CMB, dark matter initially is not repulsive. The condensed baryonic gas at the critical surface density induces dark matter repulsive force to transform dark matter in the region into repulsive dark matter repulsing one another, corresponding to the Farnes' repulsive dark matter. In this way, a galaxy actually started with a dark matter core-cusp without repulsive dark matter. The removal of the core cusp by repulsive dark matter afterward solves many difficult problems in protogalaxy and galaxy evolutions.

Protogalaxy evolution consists of six steps: 1) small core-cusp protogalaxy (BM-DM core cusp-halo), 2) large core-cusp protogalaxy (BM-DM core cusp-halo), 3) streaming protogalaxy (streaming BM core-static BM shell-DM halo), 4) SMBH protogalaxy (central SMBH-streaming BM core-static BM shell-DM halo), 5) bulge protogalaxy (central SMBH-bulge-streaming BM core-static BM 
shell-DM halo), and 6) protogalaxy termination (central SMBH-bulge-combined BM shell-DM halo) as Figure 5. In the first two steps, dark matter did not have repulsive force, so photogalaxies were small and large protogalaxies with BM-DM core-cusp-halo. In the step 3, the condensed baryonic gas at the critical surface density induces the creation tensor for dark matter repulsive force to transform dark matter in the region into repulsive dark matter repulsing one another. Through dark matter repulsive force, repulsive dark matter in the core-cusp was ejected from the core, resulting in the protogalaxy collapse which forced baryons in the BM halo to stream inward to fill the nearly empty core to form the streaming BM core. Initially, the density in the streaming BM core was too low to form star. Later, the motions of the streaming baryons prevented forming star until the streaming BM core reached the critical mass of $10^{7}-10^{8}$ $\mathrm{M}_{\odot}$ that triggered rapid atomic cooling and catastrophic baryon collapse, resulting in the supermassive star (SMS) formation in the atomically-cooled streaming BM core. The collapse of the SMS at a few $10^{5} \mathrm{M}_{\odot}$ produced the $\mathrm{DCBH}$ for the central SMBH. The bulge was generated in the streaming BM core surrounding the SMBH afterward. During and after the protogalaxy collapse, the star formations produced different types of galaxies (elliptical, spiral, barred spiral, and irregular). Essentially, the protogalaxies with the small DM core produced elliptical galaxies, the protogalaxies with the large DM core produced spiral-barred spiral galaxies, and the protogalaxies with the very large DM core produced irregular galaxies.

According to the theoretical calculation, the calculated percentages of dark energy, dark matter, and baryonic matter are 68.6 (as an input from the observation), 26, and 5.2, respectively, in agreement with observed 68.6, 26.5, and 4.9, respectively, and dark energy started in 4.33 billion years ago in agreement with the observed $4.71 \pm 0.98$ billion years ago. In conclusion, the modified Farnes' unifying theory reinterprets the Farnes' equations, and is a unifying theory of dark energy, dark matter, and baryonic matter in the positive-negative mass universe pair. The unifying theory explains protogalaxy and galaxy evolutions in agreement with the observations.

\section{Conflicts of Interest}

The author declares no conflicts of interest regarding the publication of this paper.

\section{References}

[1] Farnes, J. (2018) Astronomy \& Astrophysics, 620, A92. https://doi.org/10.1051/0004-6361/201832898

[2] Navarro, J., Frenk, C. and White, S. (1997) The Astrophysical Journal, 490, 493-508. https://doi.org/10.1086/304888

[3] Hui, L. (2001) Physical Review Letters, 86, 3467-3470. https://doi.org/10.1103/PhysRevLett.86.3467

[4] Maeder, A., et al. (2018) Planck 2018 Results. VI. Cosmological Parameters. 
[5] Komatsu, E., et al. (2009) Astrophysics Journal Supplement, 180, 330.

[6] Planck Collaboration (2016) Astronomy Astrophysics, 594, A13.

[7] Freese, K. (2017) International Journal of Modern Physics D, 26, Article ID: 1730012. https://doi.org/10.1142/S0218271817300129

[8] Chung, D. and Krasnoholovets, V. (2013) Journal of Modern Physics, 4, 77-84. https://doi.org/10.4236/jmp.2013.47A1009

[9] Chung, D. (2018) Journal of Modern Physics, 9, 2257-2273. https://doi.org/10.4236/jmp.2018.913142

[10] Chung, D. (2019) Journal of Modern Physics, 10, 1310-1341. https://doi.org/10.4236/jmp.2019.1011087

[11] Randall, L. and Sundrum, R. (1999) Physical Review Letters, 83, 3370-3373. https://doi.org/10.1103/PhysRevLett.83.3370

[12] Randall, L. (2005) Warped Passages: Unraveling the Mysteries of the Universe's Hidden Dimensions. Harper Collins, New York.

[13] Milgrom, M. (1989) Astronomy and Astrophysics, 211, 37-40.

[14] Milgrom, M. (2009) Monthly Notices of the Royal Astronomical Society, 398, 1023-1026. https://doi.org/10.1111/j.1365-2966.2009.15255.x

[15] Tanabashi, M., et al. (2019) Physical Review D, 98, Article ID: 030001.

[16] Riess, A.G., et al. (2004) Astrophysical Journal, 607, 665-687. https://doi.org/10.1086/383612

[17] De Blok, W. (2010) Advance Astronomy, 2010, Article ID: 789293. https://doi.org/10.1155/2010/789293

[18] Holmes, R.B. (2020) Journal of Modern Physics, 11, 648-667. https://doi.org/10.4236/jmp.2020.115042

[19] Chung, D. (2016) Journal of Modern Physics, 7, 642-655. https://doi.org/10.4236/jmp.2016.77064

[20] Chung, D. (2016) Journal of Modern Physics, 7, 1210-1227. https://doi.org/10.4236/jmp.2016.710110

[21] Moffat, J. (1993) International Journal of Modern Physics D, 2, 351-359. https://doi.org/10.1142/S0218271893000246

[22] Albrecht, A. and Magueijo (1999) Physics Review D, 59, Article ID: 043516. https://doi.org/10.1103/PhysRevD.59.043516

[23] Barrow, J. (2003) Physics Letters B, 564, 1-7. https://doi.org/10.1016/S0370-2693(03)00573-2

[24] Weinberg, S. (1989) Review Modern Physics, 61, 1-23. https://doi.org/10.1103/RevModPhys.61.1

[25] Chung, D. (2018) Journal of Modern Physics, 9, 2308-2319. https://doi.org/10.4236/jmp.2018.913146

[26] Gorham, P., et al. (2016) Physical Review Letters, 117, Article ID: 071101.

[27] Gorham, P., et al. (2018) Physical Review Letters, 121, Article ID: 161102.

[28] Gorham, P., et al. (2009) Astroparticle Physics, 32, 10-41.

[29] Bounias, M. and Krasnoholovets, V. (2003) The International Journal of Systems and Cybernetics, 32, 945-975. https://doi.org/10.1108/03684920310483126

[30] Chung, D. (2018) Journal of Modern Physics, 9, 2638-2656.

https://doi.org/10.4236/jmp.2018.914164 
[31] Aprile, E., et al. (2020) Observation of Excess Electronic Recoil Events in XENON1T.

[32] Fujita, J. and Miyazawa, H. (1957) Progress of Theoretical Physics, 17, 360. https://doi.org/10.1143/PTP.17.360

[33] Chung, D. (2016) Journal of Modern Physics, 7, 1150-1159. https://doi.org/10.4236/jmp.2016.710104

[34] Tsui, D., Stormer, H. and Gossard, A. (1982) Physical Review Letters, 48, 1559-1562. https://doi.org/10.1103/PhysRevLett.48.1559

[35] Stormer, H. (1999) Reviews of Modern Physics, 71, 875-889. https://doi.org/10.1103/RevModPhys.71.875

[36] Laughlin, R. (1983) Physical Review Letters, 50, 1395-1398. https://doi.org/10.1103/PhysRevLett.50.1395

[37] Loureiro, A., et al. (2019) Physical Review Letters, 123, Article ID: 081301. https://doi.org/10.1103/PhysRevLett.123.081301

[38] Steigman, G. (2007) Annual Review of Nuclear and Particle Science, 57, 463-491. https://doi.org/10.1146/annurev.nucl.56.080805.140437

[39] Chung, D. (2014) Journal of Modern Physics, 5, 1234-1243. https://doi.org/10.4236/jmp.2014.514123

[40] Chung, D. (2016) Journal of Modern Physics, 7, 1591-1606. https://doi.org/10.4236/jmp.2016.712144

[41] Bañados, E., et al. (2018) Nature, 553, 473-476. https://doi.org/10.1038/nature25180

[42] Woods, T., et al. (2018) Titans of the Early Universe: The Prato Statement on the Origin of the First Supermassive Black Holes.

[43] Neeleman, M., Prochaska, J., Kanekar, N. and Rafelski, M. (2020) Nature, 581, 269-272. https://doi.org/10.1038/s41586-020-2276-y

[44] Fall, S. and Efstathiou, G. (1980) Monthly Notices of the Royal Astronomical Society, 193, 189-206. https://doi.org/10.1093/mnras/193.2.189

[45] Dekel, A., et al. (2009) Nature, 457, 451-454. https://doi.org/10.1038/nature07648

[46] Haiman, Z., Thoul, A.A. and Loeb, A. (1996) Astrophysical Journal, 464, 523-528. https://doi.org/10.1086/177343

[47] Spergel, D., et al. (2003) The Astrophysical Journal Supplement Series, 148, 175-194. https://doi.org/10.1086/377226

[48] Readhead, A., et al. (2004) Astrophysical Journal, 609, 498-512. https://doi.org/10.1086/421105

[49] Yoshida, N., Hosokawa, T. and Omukai, K. (2012) Progress of Theoretical and EXperimental Physics, 2012, 01A305. https://doi.org/10.1093/ptep/pts022

[50] Ibata, N., Ibata, R., Famaey, B. and Lewis, G. (2014) Nature, 511, 563-566. https://doi.org/10.1038/nature13481

[51] Hirano, S., Hosokawa, T., Yoshida, N. and Kuiper, R. (2017) Science, 357, 1375-1378. https://doi.org/10.1126/science.aai9119

[52] Schauer, A., Regan, J., Glover, S. and Klessen, R. (2017) Monthly Notices of the Royal Astronomical Society, 471, 4878-4884. https://doi.org/10.1093/mnras/stx1915

[53] Regan, J. and Haehnelt, M. (2009) Monthly Notices of the Royal Astronomical Society, 396, 343-353. https://doi.org/10.1111/j.1365-2966.2009.14579.x

[54] Latif, M., Schleicher, D., Schmidt, W. and Niemeyer, J. (2013) Monthly Notices of the Royal Astronomical Society, 432, 668-678. https://doi.org/10.1093/mnras/stt503

[55] Umeda, H., Hosokawa, T., Omukai, K. and Yoshida, N. (2016) The Astrophysical 
Journal Letters, 830, L34. https://doi.org/10.3847/2041-8205/830/2/L34

[56] Woods, T., Heger, A., Whalen, D., Haemmerlé, L. and Klessen, R. (2017) The Astrophysical Journal Letters, 842, L6. https://doi.org/10.3847/2041-8213/aa7412

[57] Haemmerlé, L., Woods, T., Klessen, R., Heger, A. and Whalen, D. (2018) The Astrophysical Journal Letters, 853, L3. https://doi.org/10.3847/2041-8213/aaa462

[58] Yue, B., Ferrara, A., Salvaterra, R., Xu, Y. and Chen, X. (2014) Monthly Notices of the Royal Astronomical Society, 440, 1263-1273.

https://doi.org/10.1093/mnras/stu351

[59] Basu, S. and Das, A. (2019) The Astrophysical Journal Letters, 879, L3. https://doi.org/10.3847/2041-8213/ab2646

[60] Silk, J. and Rees, M. (1998) Astronomy and Astrophysics, 331, L1-L4.

[61] King, A. (2003) The Astrophysical Journal, 596, L27-L29. https://doi.org/10.1086/379143

[62] Lasker, R., et al. (2017) The Astrophysical Journal, 825, 1. https://doi.org/10.3847/0004-637X/825/1/3

[63] Schramm, M. and Silverman, J. (2013) The Astrophysical Journal, 767, 13. https://doi.org/10.1088/0004-637X/767/1/13

[64] Ibata, R., et al. (2011) The Astrophysical Journal, 738, 186. https://doi.org/10.1088/0004-637X/738/2/186

[65] Lin, C. and Shu, F. (1964) Astrophysical Journal, 140, 646-655. https://doi.org/10.1086/147955

[66] Shu, F. (2016) Annual Review of Astronomy and Astrophysics, 54, 667-724. https://doi.org/10.1146/annurev-astro-081915-023426

[67] Bournaud, F. and Combes, F. (2002) Astronomy and Astrophysics, 392, 83-102. https://doi.org/10.1051/0004-6361:20020920 